\title{
Osteopontin deficiency ameliorates Alport pathology by preventing tubular metabolic deficits
}

\author{
Wen Ding,,$^{1,2}$ Keyvan Yousefi, ${ }^{1,2}$ Stefania Goncalves, ${ }^{3}$ Bradley J. Goldstein, ${ }^{2,3}$ Alfonso L. Sabater, ${ }^{4}$ \\ Amy Kloosterboer, ${ }^{5}$ Portia Ritter, ${ }^{5}$ Guerline Lambert, ${ }^{5}$ Armando J. Mendez, ${ }^{6}$ \\ and Lina A. Shehadeh ${ }^{2,5,7,8}$ \\ 'Department of Molecular and Cellular Pharmacology, ${ }^{2}$ Interdisciplinary Stem Cell Institute, ${ }^{3}$ Department of \\ Otolaryngology, ${ }^{4}$ Department of Ophthalmology, Bascom Palmer Eye Institute, ${ }^{5}$ Department of Medicine, Division of \\ Cardiology, ${ }^{6}$ Diabetes Research Institute, ${ }^{7}$ Vascular Biology Institute, and ${ }^{8}$ Peggy and Harold Katz Family Drug Discovery \\ Center, University of Miami Leonard M. Miller School of Medicine, Miami, Florida, USA.
}

\begin{abstract}
Alport syndrome is a rare hereditary renal disorder with no etiologic therapy. We found that osteopontin (OPN) is highly expressed in the renal tubules of the Alport mouse and plays a causative pathological role. OPN genetic deletion ameliorated albuminuria, hypertension, tubulointerstitial proliferation, renal apoptosis, and hearing and visual deficits in the Alport mouse. In Alport renal tubules we found extensive cholesterol accumulation and increased protein expression of dynamin-3 (DNM3) and LDL receptor (LDLR) in addition to dysmorphic mitochondria with defective bioenergetics. Increased pathological cholesterol influx was confirmed by a remarkably increased uptake of injected Dil-LDL cholesterol by Alport renal tubules, and by the improved lifespan of the Alport mice when crossed with the LdIr/- mice with defective cholesterol influx. Moreover, OPN-deficient Alport mice demonstrated significant reduction of DNM3 and LDLR expression. In human renal epithelial cells, overexpressing DNM3 resulted in elevated LDLR protein expression and defective mitochondrial respiration. Our results suggest a potentially new pathway in Alport pathology where tubular OPN causes DNM3- and LDLR-mediated enhanced cholesterol influx and impaired mitochondrial respiration.
\end{abstract}

Conflict of interest: LAS and the University of Miami filed for a patent (PCT/US17/45536: Method of Treating Alport Syndrome) in August 2017.

Submitted: April 28, 2017

Accepted: February 9, 2018

Published: March 22, 2018

Reference information: JCI Insight. 2018;3(6):e94818. https:// doi.org/10.1172/jci.insight.94818.

\section{Introduction}

Alport syndrome was first described in 1927 by Cecil A. Alport as a rare hereditary congenital hemorrhagic nephritis (1). Microscopic hematuria is the most common and earliest manifestation of the disease. Later in life, clinical implications of Alport syndrome consist of proteinuria, hypertension, progressive renal failure, high-frequency sensorineural hearing loss, and ocular anomalies. A small population also presents other symptoms including dyspnea, facial puffiness, pedal edema, mental retardation, and increased risk of cardiovascular events $(2,3)$. Mutations of type IV collagen chains, which are major components of capillary basement membranes, cause Alport syndrome. Normally, embryonic $\alpha 1 \alpha 1 \alpha 2$ chain is replaced during development by the mature $\alpha 3 \alpha 4 \alpha 5$ chain $(4,5)$. However, such a switch is absent in Alport patients, rendering glomerular basement membrane (GBM) more vulnerable to filtration pressure and endoproteolysis (6). Thickened glomerular (or other) capillary basement membrane is a major pathology of Alport patients. Despite possible early diagnosis, there is currently no therapy to prevent end-stage renal failure in Alport patients and the standard care for Alport patients is limited to antihypertensive medications and eventually dialysis and kidney transplant.

The $\mathrm{Col}_{4} 3^{--}$Alport mouse is a standard model for autosomal recessive Alport syndrome, as the mouse demonstrates many symptoms of an Alport patient (7-9). The Alport mouse gradually develops severe proteinuria, glomerulosclerosis, and tubulointerstitial fibrosis, and dies at approximately 10-24 weeks of age depending on the genetic background (10). Otologic assessments have shown that the Alport mouse tends to have elevated hearing thresholds with age, as seen in the human syndrome (7). Ocular abnormalities have not been investigated previously in the Alport mouse. Here, we further characterize the Alport mouse on mixed genetic background (C57BL6 × 129Sv/J $\times$ BALB/c) and 
show previously unreported clinically relevant phenotypes including hypertension, lenticonus, thickened retinal capillary basement membrane, dysmorphic and dysfunctional renal tubular mitochondria, and an immensely increased renal cholesterol influx.

Osteopontin (OPN) is a secreted phosphoprotein that plays important roles in regulating bone morphogenesis, inflammation, heart failure, and tumor metastasis $(11,12)$. Renal OPN mRNA and protein were increased in animal models of albuminuria and urinary OPN protein levels were elevated in children with nephrotic syndrome (13). In cultured murine podocytes, recombinant OPN protein increased the expression of matrix-degrading enzymes, and the motility of podocytes, both of which are strongly associated with the development of proteinuria (13-15). In this study, we report a potentially novel OPN-driven signaling pathway that plays a putatively central role in Alport pathology. We show that OPN is highly expressed in Alport renal tubules where it regulates dynamin-3-mediated (DNM3-mediated) increased LDL receptor (LDLR) expression, increased LDL-cholesterol influx, and decreased mitochondrial energetics.

\section{Results}

OPN is substantially expressed in the tubules of $\mathrm{Col}_{4 a 3^{--}}$mice. To characterize the role of OPN in Alport syndrome, we studied OPN expression by immunostaining and Western blots in kidney and/or plasma samples from wildtype and $\mathrm{Col}_{4} 3^{--1}$ mice. By costaining for OPN and the podocyte protein synaptopodin (SYNPO), we found substantially elevated OPN expression in the renal tubules of $\mathrm{Col}_{4} \mathrm{3}^{-1-}$ mice (Figure 1A). Western blots revealed a dramatic increase of OPN expression in kidneys (Figure 1B) and plasma (Figure 1C) of $\mathrm{Col}_{43^{-1}}$ mice.

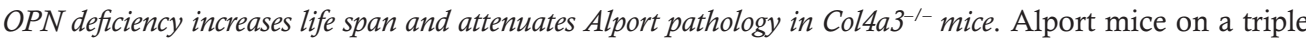
mixed background develop severe renal dysfunction and die approximately at 10 weeks of age. In order to test whether OPN deficiency increases lifespan in Alport mice, we recorded survival of $\mathrm{Col}_{4} \mathrm{a}^{-1-}, \mathrm{Col}_{4} \mathrm{a}^{-1-}$ $\mathrm{Opn}^{+/-}$, and $\mathrm{Col}_{4 a 3^{--}} \mathrm{Opn}^{-/-}$animals and monitored the body weights at 9-12 weeks of age. Kaplan-Meier survival curves show that the $\mathrm{Col}_{4} \mathrm{a3}^{-/-} \mathrm{Opn}^{-/-}$and $\mathrm{Col}_{4} \mathrm{a}^{-/-} \mathrm{Opn}^{+/-}$mice significantly outlived the Alport mice (Figure 2A). In addition, the body weights were significantly increased in OPN-deficient animals (Figure 2B). Impressively, $2 \mathrm{Col}_{4} \mathrm{a}^{-/-} \mathrm{Opn}^{+/-}$mice lived up to 20 weeks (Figure 2A), doubling the lifespan of Alport animals. To study the functional effects of OPN deficiency on renal pathology, urine and blood samples from animals at 8-9 weeks were analyzed. At 8-9 weeks of age, Alport mice develop severe proteinuria, renal dysfunction, hearing deficits, and eye abnormalities. Urinary albumin/creatinine levels were markedly increased in Alport animals by 161 times compared with wild type (as expected), and significantly decreased by $50 \%$ in $\mathrm{Col}_{4 a 3^{-/-}} \mathrm{Opn}^{+/-}$and $\mathrm{Col}_{4} \mathrm{a}^{-/-} \mathrm{Opn}^{-/-}$mice as compared with $\mathrm{Col}_{43^{-/-}}$mice (Figure 2C). We also assessed other renal dysfunction parameters like plasma blood urea nitrogen (BUN) and creatinine (CRE), and found a modest but not statistically significant decrease in $\mathrm{Col}_{43^{-/-}} \mathrm{Opn}^{+/-}$and $\mathrm{Col}_{4} \mathrm{a}^{-1-} \mathrm{Opn}^{-/-}$mice as compared with $\mathrm{Col}_{4} \mathrm{a3}^{-/-}$mice (Figure 2, D and E). We show that the blood mean

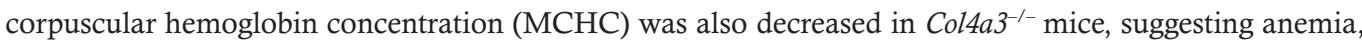
which is often seen in patients with chronic kidney disease (16) and rarely in X-linked Alport syndrome (17). Our data show that OPN deficiency significantly improved MCHC in Alport mice (Figure 2F). Interestingly, we found galectin-3, whose expression correlates with end-stage renal dysfunction (ESRD) (18), was expressed 2 times higher in $\mathrm{Col}_{4} \mathrm{a}^{-/-}$mice than wild-type controls and significantly decreased to nor-

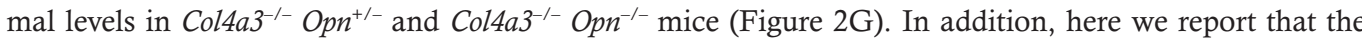
$\mathrm{Col}_{4} \mathrm{a}^{-/-}$mice developed moderate hypertension relative to wild-type mice and that the hypertension was markedly reduced in $\mathrm{Col}_{4} \mathrm{a3}^{-/-} \mathrm{Opn}^{-/-}$and $\mathrm{Col}_{4} 3^{-/-} \mathrm{Opn}^{+/-}$mice (Figure 2, $\mathrm{H}$ and I). Our data show that OPN deficiency reduced high blood pressure, which is a major and early pathology in Alport patients.

OPN deficiency tends to improve high-frequency hearing threshold and reduces cochlear pathology in Col4a $3^{-1-}$ mice. Otopathology of Alport syndrome is characterized by high-frequency sensorineural hearing loss and this otopathology is present in the Alport mouse (8). On the other hand, thickened cochlear strial capillary basement membrane has been reported in the Alport mouse (8) but not in Alport patients (19-22). To examine the effects of OPN deficiency on hearing ability of Alport mice, we recorded the responses to a series of

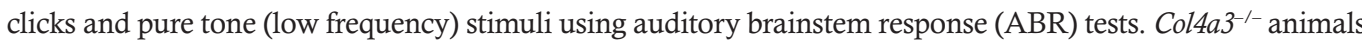
showed diminished responses to clicks and pure tone stimuli. Interestingly, we found that $\mathrm{Col}_{43^{-/-}} \mathrm{Opn}^{+/-}$ mice showed tendency for improved hearing ability in response to 8 - and $16-\mathrm{kHz}$ pure tones and to click stimuli (Figure 3A) as compared with Alport mice. This result was not seen on the OPN-null background. However, the striking morphological changes of thickened capillary basement membrane surrounding the stria vascularis of $\mathrm{Col}_{4} \mathrm{a}^{-1-}$ mice were ameliorated in the cochlea of $\mathrm{Col}_{43^{-1-}} \mathrm{Opn}^{-1-}$ mice (Figure 3B). We had 
A
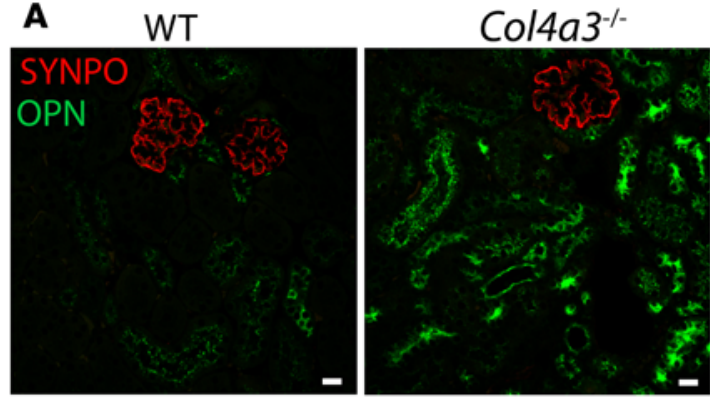

Kidney

B

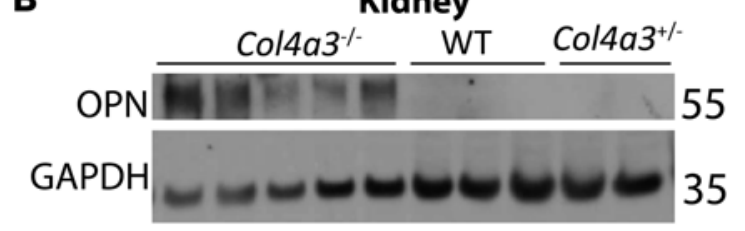

C

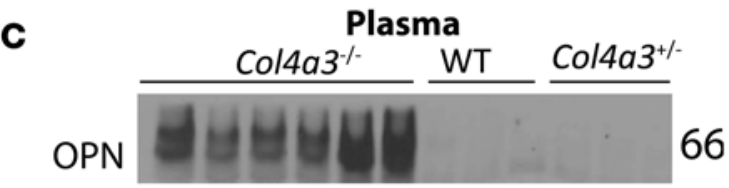

Ponceau
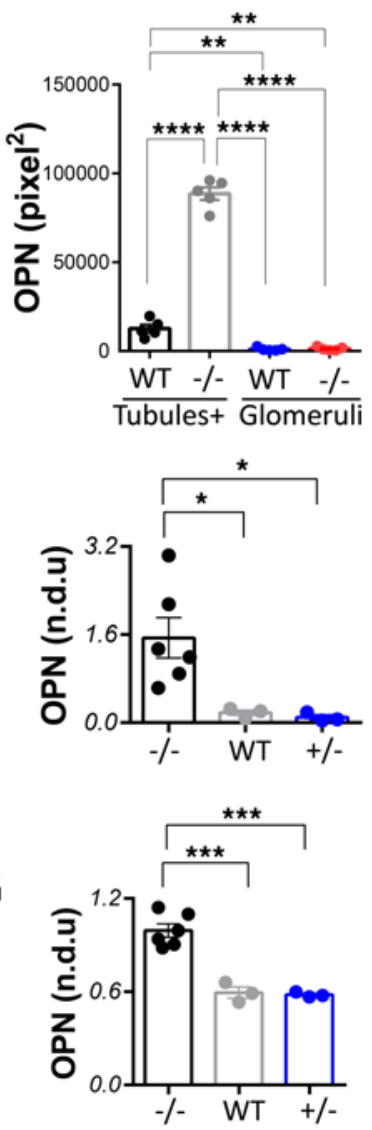

Figure 1. OPN expression is increased in Alport mice. (A) Osteopontin (OPN) expression is substantially elevated in the renal tubules of Alport versus wild-type (WT) mice as shown by immunostaining and confocal microscopy. Tubules+ stands for quantifications of OPN staining in tubules and peritubular capillaries. Images are representative of 6 mice per group. Scale bars: $20 \mu \mathrm{m}$. Western blots and corresponding densitometry show elevated OPN expression in $\mathrm{Col}_{4 a 3^{-/}}$ versus WT or $\mathrm{Col}_{4} \mathrm{a}^{+/-}$mouse kidneys (B) and plasma (C). $n=3-6$ mice per group. Quantification is based on 3 independent experiments. Data are the mean \pm SEM. ${ }^{*} P<0.05$, ${ }^{* *} P<0.01,{ }^{* *} P<0.001,{ }^{* * *} P<$ 0.0001 based on ANOVA with Tukey's post hoc test. n.d.u., normalized densitometry units.

shown in a model of heart failure that while homozygous loss of OPN may be beneficial in the short term, it is deleterious in the long term (23). In the Alport model, mice die very early and we found that homozygous loss of OPN was as beneficial as heterozygous loss for most endpoints except for the hearing function. We think that the hearing data are more realistic and that heterozygous deficiency or blockade of OPN is more physiological and beneficial. The lack of statistical significance in the protective trend in the Alport with OPN heterozygous loss is likely due to the severity of the disease in the Alport mouse model on triple background. Testing the hearing function with OPN deficiency in the more resilient Alport mouse on C57BL6 background $(10,24)$ will not be helpful either, as C57BL6 mice lose hearing senses early in life $(25,26)$.

OPN deficiency reduces ocular pathology in $\mathrm{Col}_{4} 3^{-1-}$ mice. Ocular manifestations in Alport patients include corneal opacities, anterior lenticonus, and retinopathy (27). To characterize corneal and lens abnormalities in Alport mice, we performed optical coherence tomography (OCT) studies to visualize anterior segment structures of the eye, as shown in the schematic in Figure 4A. We analyzed the central corneal thickness (CCT) and anterior chamber depth (ACD) and did not observe a difference among the various genotypes (Figure 4, B and C). Next we examined the anterior capsule apical angle (ACAA), the decrease of which represents conical protrusion of lens to the anterior segment and is used to describe anterior lenticonus in Alport patients (28). Our analysis showed that the ACAA was significantly decreased in $\mathrm{Col}_{4} \mathrm{a}^{-/-}$mice and

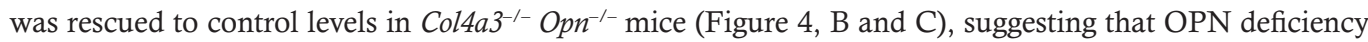
reduced anterior lenticonus developed in $\mathrm{Col}_{4} \mathrm{a}^{-/-}$mice. In addition, electron microscopy (EM) revealed a dramatic thickening of the basement membranes in the retinal capillaries, and that was restored in OPNdeficient Alport mice (Figure 4D). These findings suggest that the Alport mice develop lenticonus and retinal capillary basement membrane thickening that are rescued by OPN deficiency.

OPN deficiency reduces renal injury, tubular cell proliferation, lipid accumulation, GBM thickness, and DNM3 expression in Col4a3 ${ }^{-1-}$ mice. Kidney injury molecule-1 (KIM-1), a type 1 transmembrane protein whose expression is markedly upregulated in the proximal tubules in acute tubular necrosis in

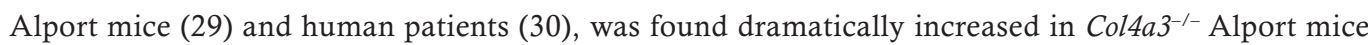

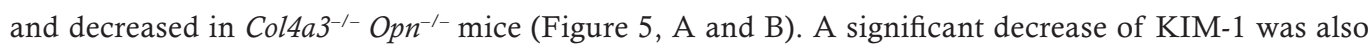
observed in $\mathrm{Col}_{43^{-/-}} \mathrm{Opn}^{+/-}$mice (fold change $[\mathrm{FC}]=0.41, P=0.05$ ) as compared with the Alport, 
A
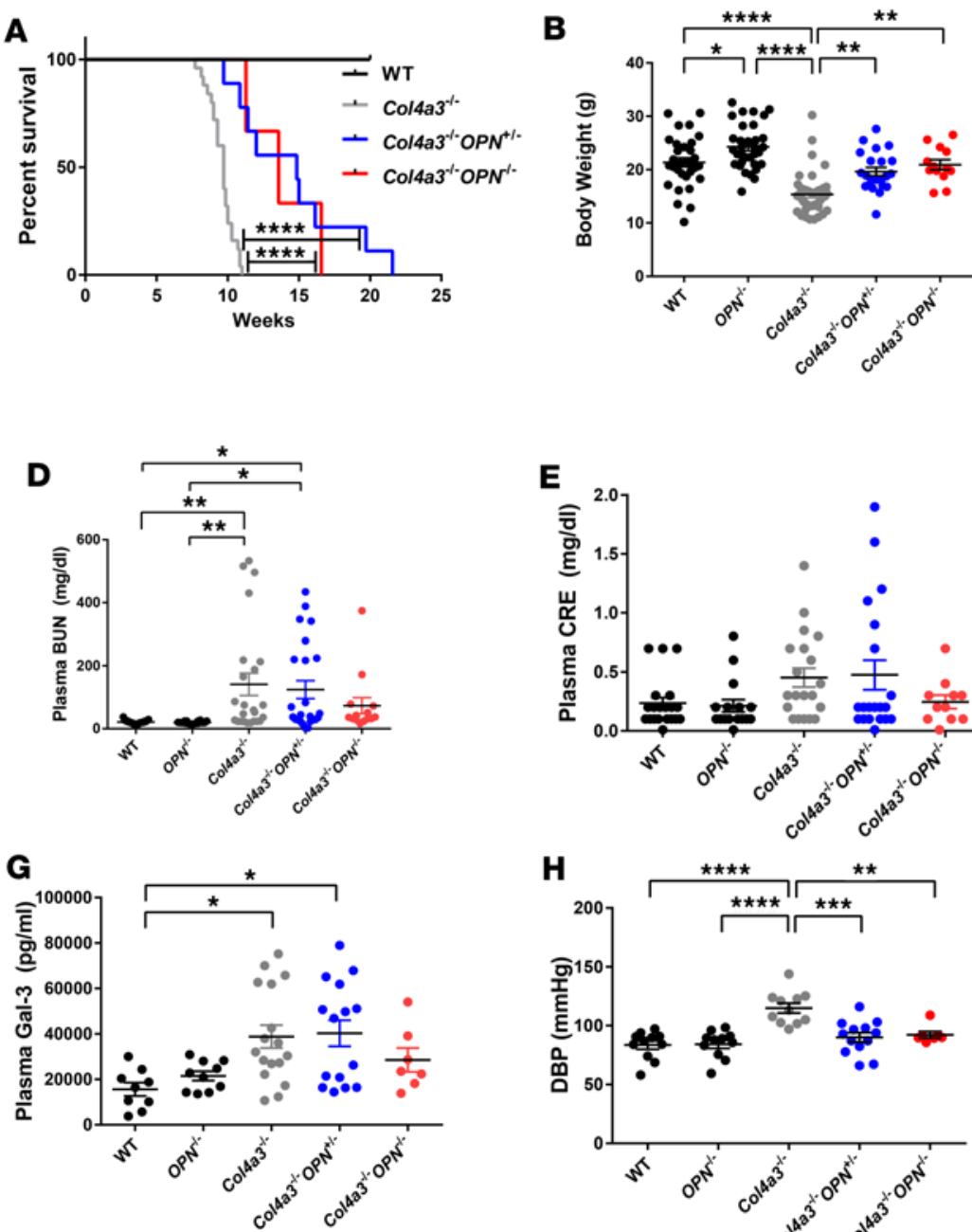

B
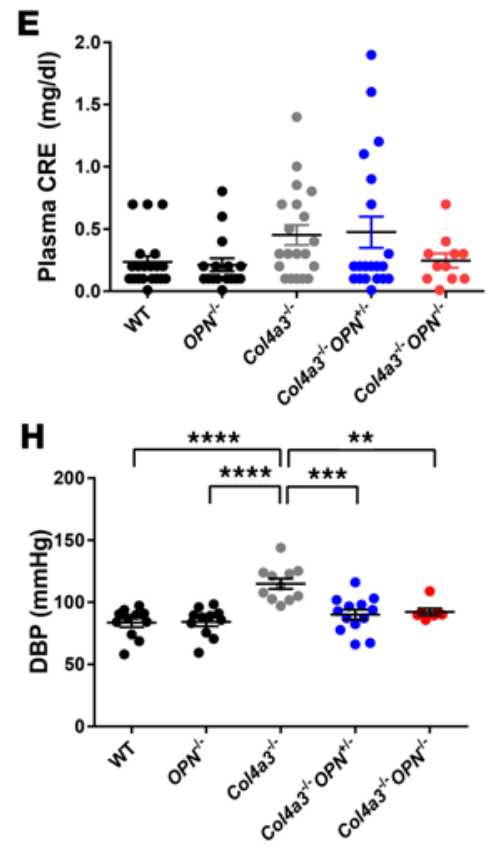

C

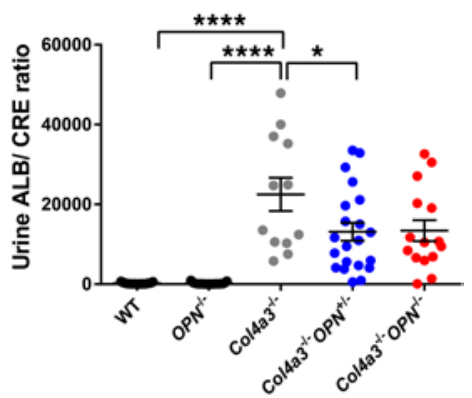

F

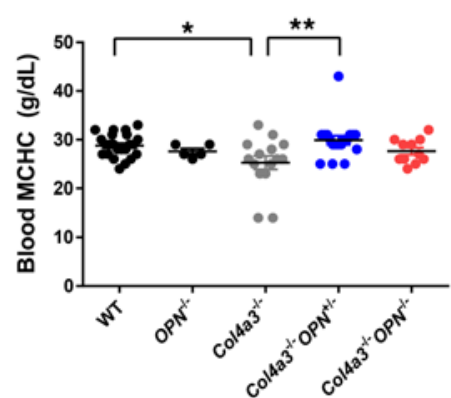

I

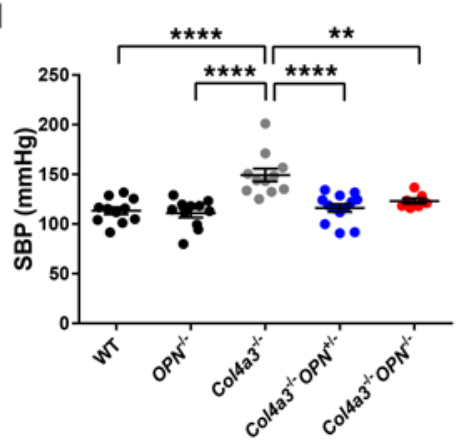

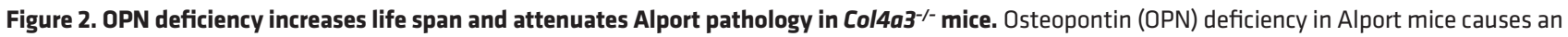

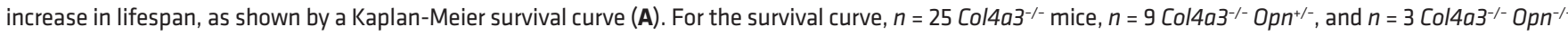
mice. OPN deficiency in Alport mice causes a reduction in body weight loss (B), a reduction of albuminuria as measured by albumin (ALB) and creatinine (CRE) ELISA (C), a decrease in plasma blood urea nitrogen (BUN) (D), and CRE (E) levels, an increase in blood mean corpuscular hemoglobin concentration (MCHC) $(\mathbf{F})$, a reduction in plasma galectin-3 (Gal-3) as measured by ELISA (G), and reductions in diastolic (DBP) and systolic blood pressure (SBP) as recorded by tailcuff blood pressure system ( $\mathbf{H}$ and $\mathbf{I})-$ all relative to Alport mice. Data were collected from animals at $8-9$ weeks of age. Data are the mean $\pm S E M$. ${ }^{*} P<0.05$, ${ }^{* *} P<0.01,{ }^{* *} P<0.001,{ }^{* * *} P<0.0001$ based on ANOVA with Tukey's post hoc test. Detailed statistics are shown in Supplemental Table 2.

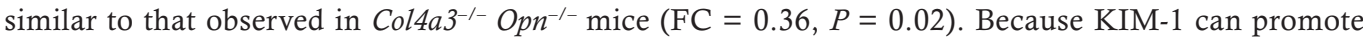
renal fibrosis (31), decreased KIM-1 in $\mathrm{Col}_{4 a 3^{-/-}} \mathrm{Opn}^{-/-}$mice suggested an antifibrotic effect for OPN deficiency in Alport mice. To directly test fibrotic proliferation, daily 5-ethynyl-2'-deoxyuridine (EdU) injections were performed for 30 consecutive days in Alport mice. EdU incorporation was examined in histological kidney sections that were costained for SYNPO. Our data showed that the majority of EdU was present in tubules and interstitial space. In $\mathrm{Col}_{43^{-/-}}$mice, extensive EdU incorporation was

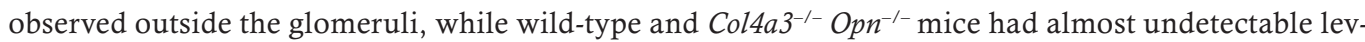
els of EdU (Figure 5, C and D). These data suggest that OPN expression in the injured Alport tubules is correlated with high tubular fibrotic proliferation.

Dysregulated lipid metabolism has been implicated in fibrosis of various tissues $(32,33)$, in nephrotic glomeruli (34-36), and in Alport nephropathy (37). To investigate dysregulated lipid accumulation in Alport mice, using Oil Red, we stained for lipids in frozen kidney sections collected from 3 genotypes. Impressively, we found extensive lipid deposition on tubules and in interstitial spaces in $\mathrm{Col}_{4} \mathrm{a}^{-1-}$ mice,

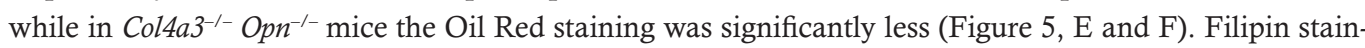


A
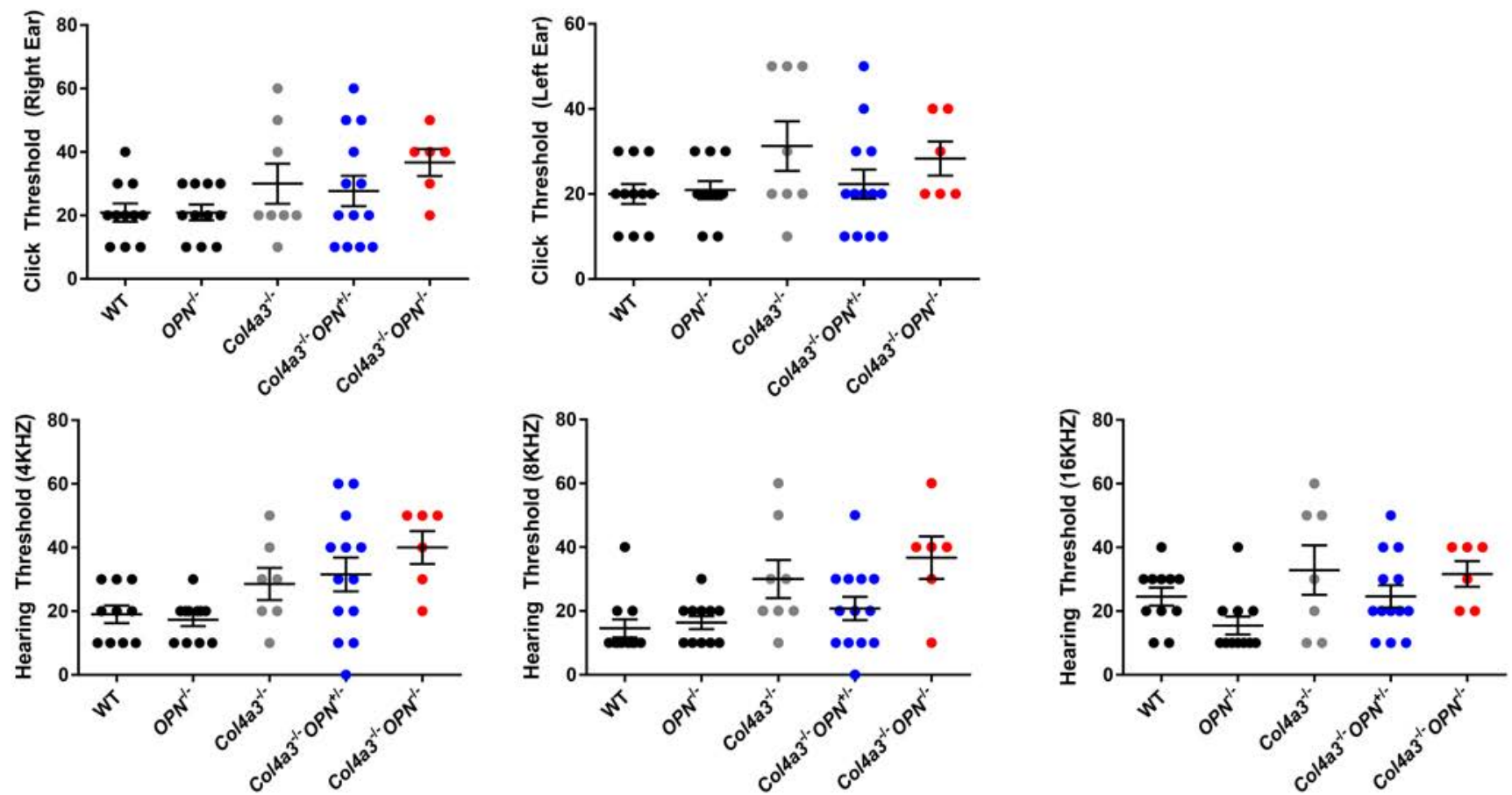

B

WT

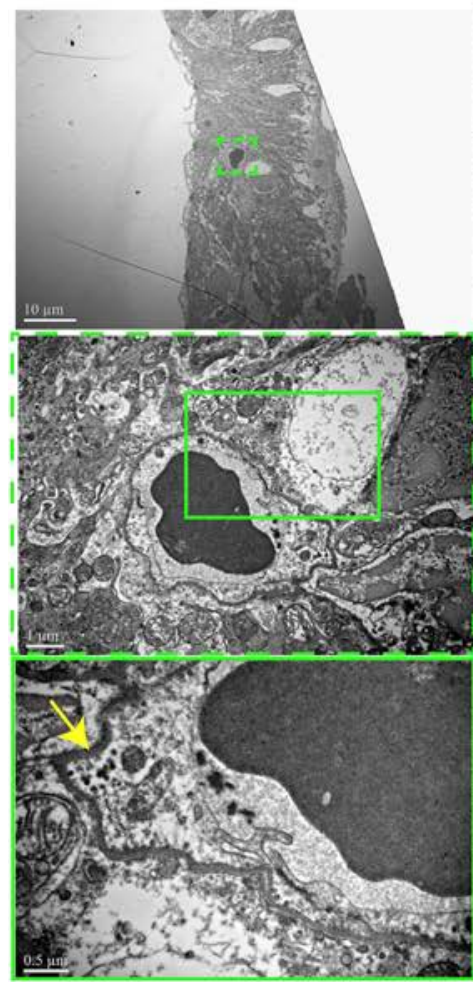

$\mathrm{Col} 4 \mathrm{a}^{\%}$
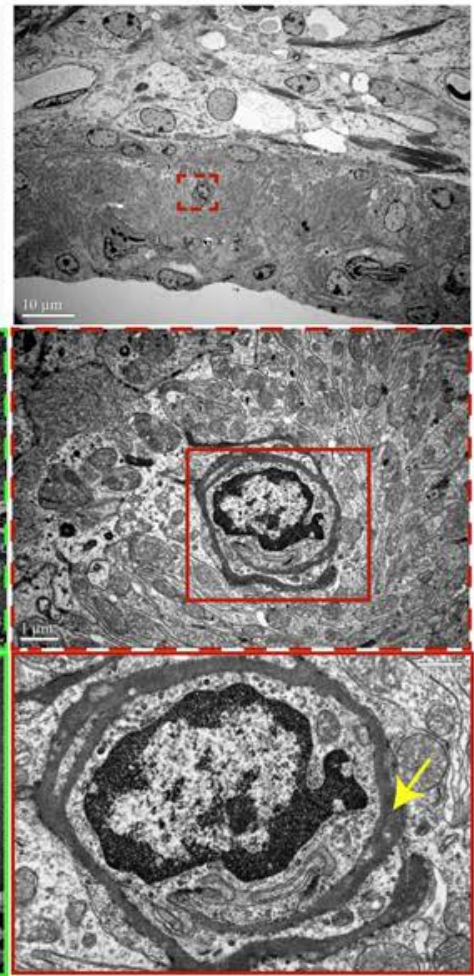

$\mathrm{Col} 4 \mathrm{ar}^{\%} \mathrm{Opn} \%$
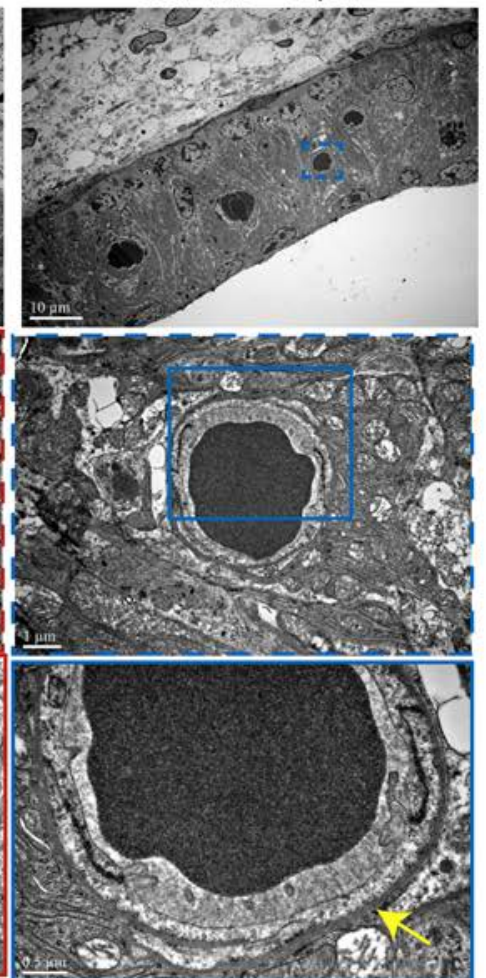

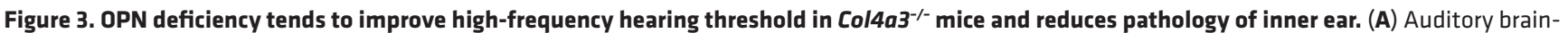
stem response (ABR) was performed for recording hearing thresholds across click stimuli (top panel) or pure tone stimuli at different frequencies (bottom panel). Ostepontin (OPN) deficiency suggests an improvement (though nonsignificant based on ANOVA with Tukey's post hoc test) of hearing ability of Alport mice at the $16-\mathrm{kHZ}$ stimuli. $n=6-12$ mice per group. Data are the mean \pm SEM. (B) Representative electron microscopy (EM) images show thickened basement membrane in Alport cochleas - a pathology that is reduced with OPN deficiency. Insets are shown at a higher magnification on the bottom. Yellow arrows point to basement membrane. 
A

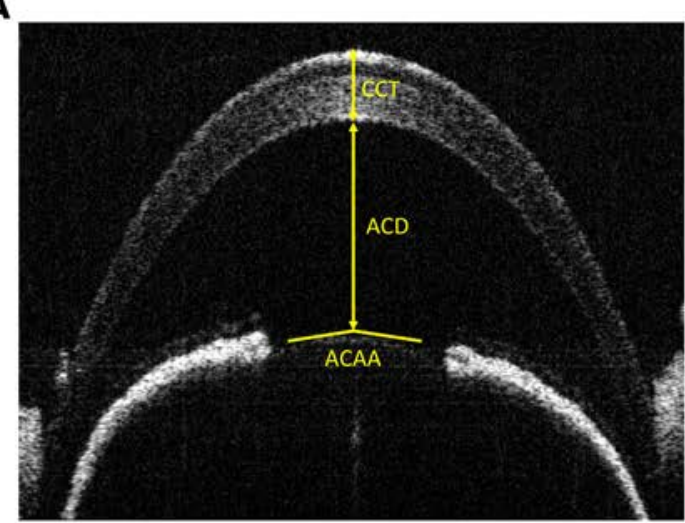

B

B WT

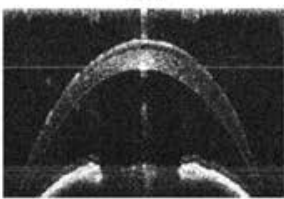

D
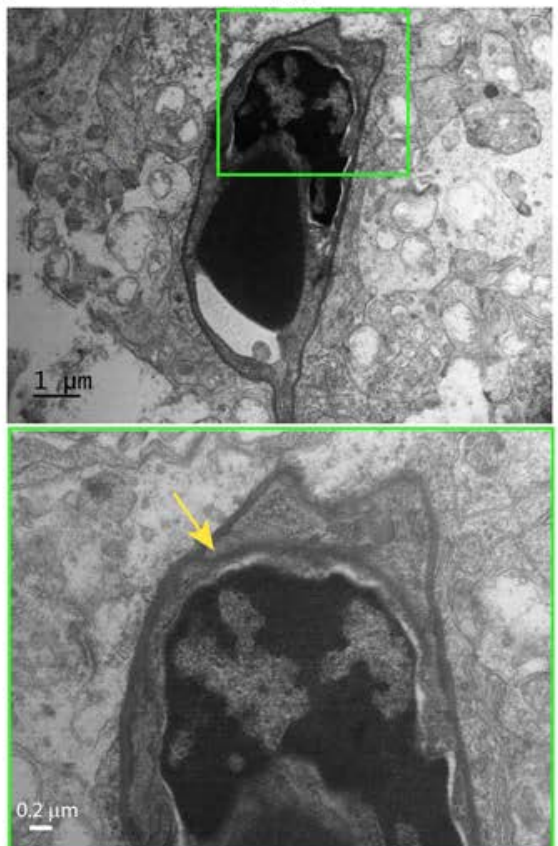

C

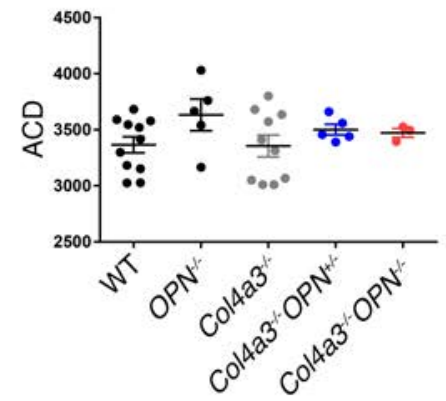

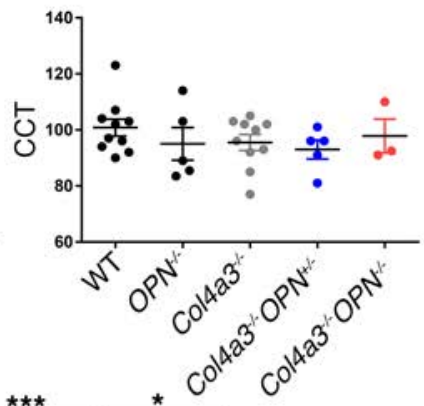

\section{$\mathrm{Col} 4 \mathrm{ar}^{\%}$}
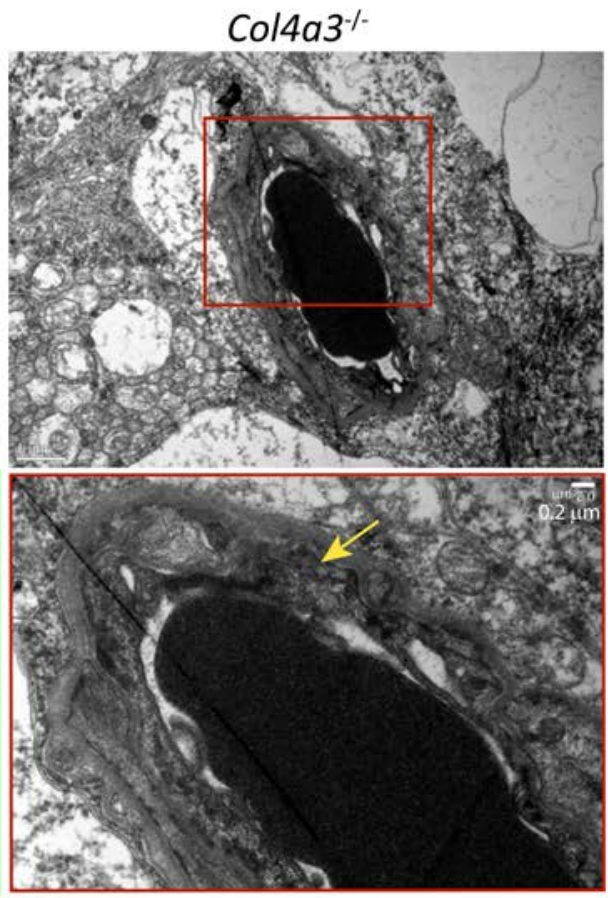

\section{Col4a3\% Opn $\%$}

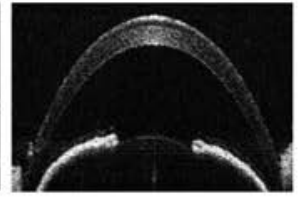

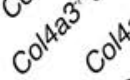

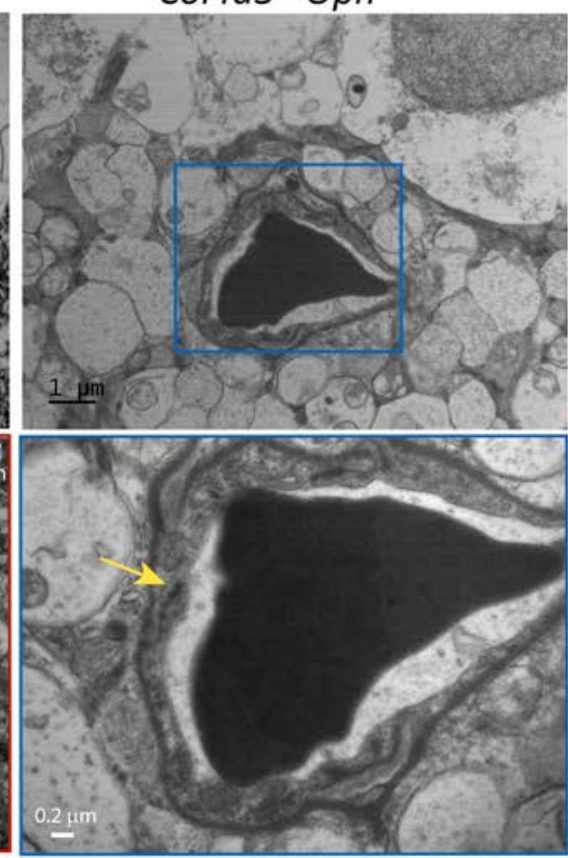

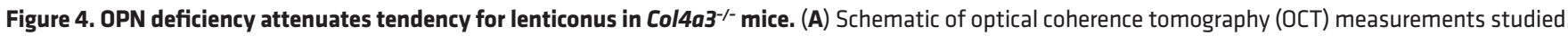
ACD, anterior chamber depth; CCT, central corneal thickness; ACAA, anterior capsule apical angle. (B and C) OCT measurements show that ACD and CCT are unaltered with OPN deficiency, while ACAA is significantly increased in Alport osteopontin-deficient (OPN-deficient) mice relative to Alport mice. $n=3-10$ mice per group. Data are the mean $\pm \mathrm{SEM} .{ }^{*} P<0.05$, ${ }^{* *} P<0.001$, ${ }^{* * *} P<0.0001$ based on ANOVA with Tukey's post hoc test. (D) Electron microscopy shows

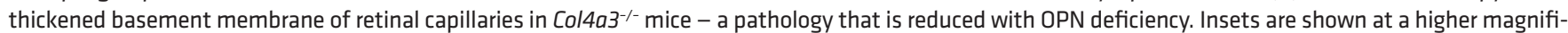
cation on the bottom. Yellow arrows point to basement membrane.

ing revealed a dramatic increase of free cholesterol deposits in the renal tubules of $\mathrm{Col}_{4} \mathrm{3}^{-1-}$ mice, which was markedly reduced in $\mathrm{Col}_{43^{-/-}} \mathrm{Opn}^{-/-}$mice (Supplemental Figure 1A; supplemental material available online with this article; https://doi.org/10.1172/jci.insight.94818DS1). In addition, we quantified triglycerides in whole-kidney lysates. Interestingly, there was no increase in total kidney triglycerides in Alport mice compared with wild type (Supplemental Figure 1B). 
EM and corresponding quantification showed dramatically thickened GBM and effacement of podo-

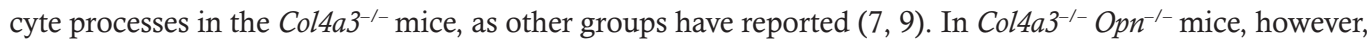
the thickness of the GBM and podocyte attachment were significantly improved (Figure 5, G and $\mathrm{H}$ ).

Because OPN regulates macrophage function (38), and interstitial inflammation is a key feature of progressive renal dysfunction including that in Alport syndrome $(39,40)$, we investigated macrophage infiltration by staining for CD68, which has been reported in foam cells in Alport patients (41). Quantification of the CD68 macrophage marker showed that there is no reduction of macrophage infiltration in the Alport kidneys on the OPN-knockout background (Supplemental Figure 2A), suggesting that suppressing inflammatory responses is not the mechanism by which OPN deficiency reduces Alport pathology. Similarly, we studied apoptosis in Alport mice with or without OPN deficiency (42). TUNEL staining showed that apoptosis was less prominent in the OPN-deficient Alport kidneys (Supplemental Figure 2B)

Moreover, to elucidate the mechanism by which OPN reduces renal pathology in Alport animals in an

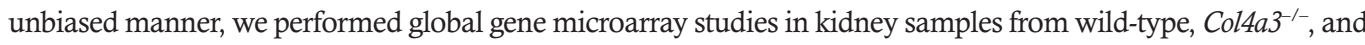
$\mathrm{Col}_{4 \mathrm{a}^{-1-}} \mathrm{Opn}^{-1-}$ mice. We found that 11 genes were upregulated and 4 genes were downregulated in Alport relative to wild-type kidneys, and normalized by OPN deficiency (Supplemental Table 1). Interestingly, we found that Dnm3, a member of the enzymatic GTPase family that regulates clathrin vesicle-mediated cholesterol endocytosis (43), was significantly upregulated in $\mathrm{Col}_{4} 3^{-/-}$mice and downregulated in $\mathrm{Col}_{43^{-1-}} \mathrm{Opn}^{-/-}$mice (Supplemental Table 1). Other dynamin genes including Dnm2 and Dnm1 showed less dramatic expression patterns. Dnm2 transcripts were increased in $\mathrm{Col}_{4} \mathrm{a}^{-1-}$ mice relative to wild-type control, and remained high in

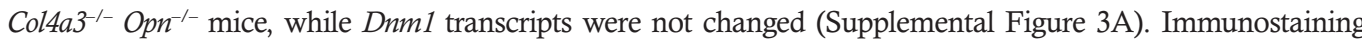
further confirmed that DNM2 protein levels were high among the Alport genotypes (Supplemental Figure 4). To validate the expression pattern of DNM3, we performed immunohistochemistry on kidney sections and found that the DNM3 protein level was elevated in the tubules of $\mathrm{Col}_{4} \mathrm{a}^{-1-}$ mice and normalized in the $\mathrm{Col}_{4 a 3^{--}} \mathrm{Opn}^{-1-}$ mice (Figure 5I). This was further confirmed by Western blot quantification (Figure 5J).

$D N M 3$ is positively regulated by OPN and contributes to cholesterol influx in alport renal tubules though LDLR upregulation. Since our results showed a reduction of DNM3 RNA and protein levels in OPN-deficient Alport mouse kidneys, we then examined if OPN protein directly induces DNM3 expression in vitro in tubular epithelial cells. As TGF- $\beta 1$ participates in key events in Alport renal disease progression $(44,45)$, we treated human renal epithelial HK-2 cells daily with $5 \mathrm{ng} / \mathrm{ml}$ recombinant TGF- $\beta$ protein to induce stress. Recombinant OPN $(1 \mu \mathrm{g} / \mathrm{ml})$ protein was added to the stressed cells every 24 hours, and the cells were collected 48 hours after the first OPN treatment. We found that DNM3 protein levels were significantly increased in OPN-treated HK-2 cells (Figure 6, A and B).

Since DNM3 is a member of enzymatic GTPase family that regulates clathrin vesicle-mediated cholesterol endocytosis (43), we hypothesized that the negative regulation of lipid accumulation by OPN is via DNM3-mediated cholesterol endocytosis. To directly test this hypothesis, we infected 293T and HK-2 cells with a CMV-DNM3 or scrambled (SCR) control lentivirus and validated by quantitative PCR (qPCR) the overexpression of DNM3 transcripts in both stable cell lines (Figure 6C). Most interestingly, we found that overexpression of DNM3 in HK-2 cells caused a significant increase in LDLR protein levels (Figure $6, \mathrm{D}$ and E). Conversely, inhibition of dynamins by dynasore, known to decrease dynamins and increase PAI-1 proteins (46), caused a significant decrease of LDLR and increase of PAI-1 protein levels in HK-2 cells (Figure 6F). Similarly, dynasore treatment caused a significance decrease in LDLR protein levels in mouse primary renal epithelial cells isolated from wild-type or Alport mice (Figure 6G). We then validated by immunostaining in Alport mice on OPN-positive or -negative backgrounds that LDLR was dramatically increased in Alport renal tubules and significantly decreased in $\mathrm{Col}_{4 a 3^{-/-}} \mathrm{Opn}^{-/-}$and $\mathrm{Col}_{43^{3--}} \mathrm{Opn}^{+/-}$renal tubules (Figure 6, H and I). However, Ldlr transcripts were not changed in the Alport kidneys according to microarrays (Supplemental Figure 3B), suggesting that OPN likely regulates LDLR at the posttranscriptional level. To validate increased cholesterol influx in Alport kidneys, Alport or wild-type mice were injected with $150 \mu \mathrm{g}$ of DiI-LDL for 2 hours, and kidneys and livers were studied histologically. We found a remarkable 45-fold increase in DiI-LDL influx in the Alport renal tubules relative to the wild-type group (Figure 6J), suggesting that this abnormal rate of cholesterol influx in the renal tubules of Alport mice may be the reason for the observed tubular lipid accumulation described above (Figure 5, E and F). Interestingly, a mild yet significant increase of DiI-LDL levels was observed in the Alport livers relative to wild-type livers (Supplemental Figure 5). To validate this observation, we crossed the Alport mice with LDLR-knockout mice that have defective cholesterol influx even with heterozygous deletion (47) and generated $\mathrm{Col}_{4 a 3^{-1}}$ 
A

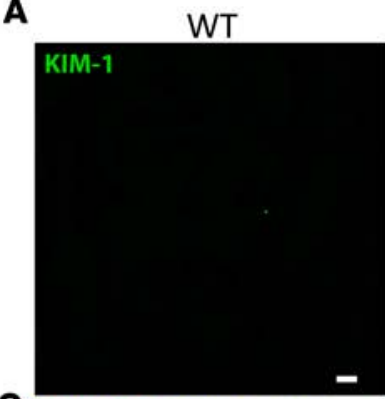

c
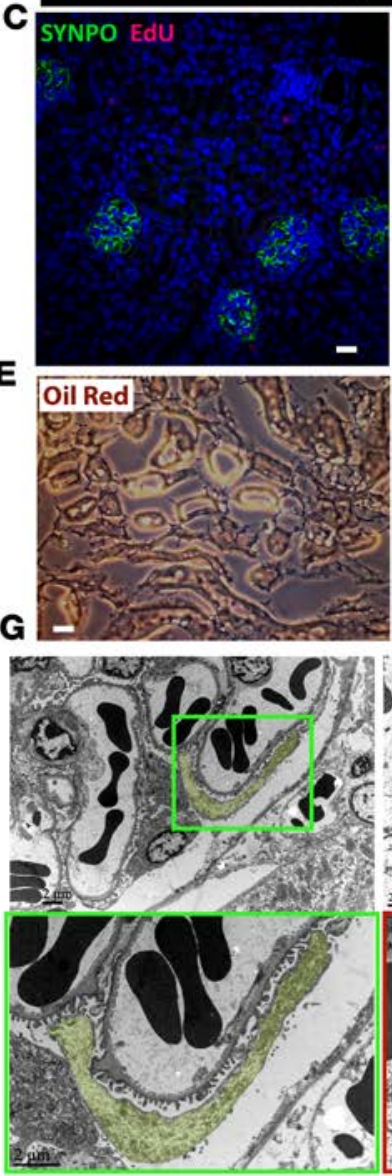

I DNM3

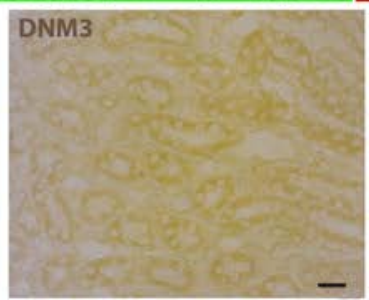

$\mathrm{Col} 4 \mathrm{a}^{\%} \%$
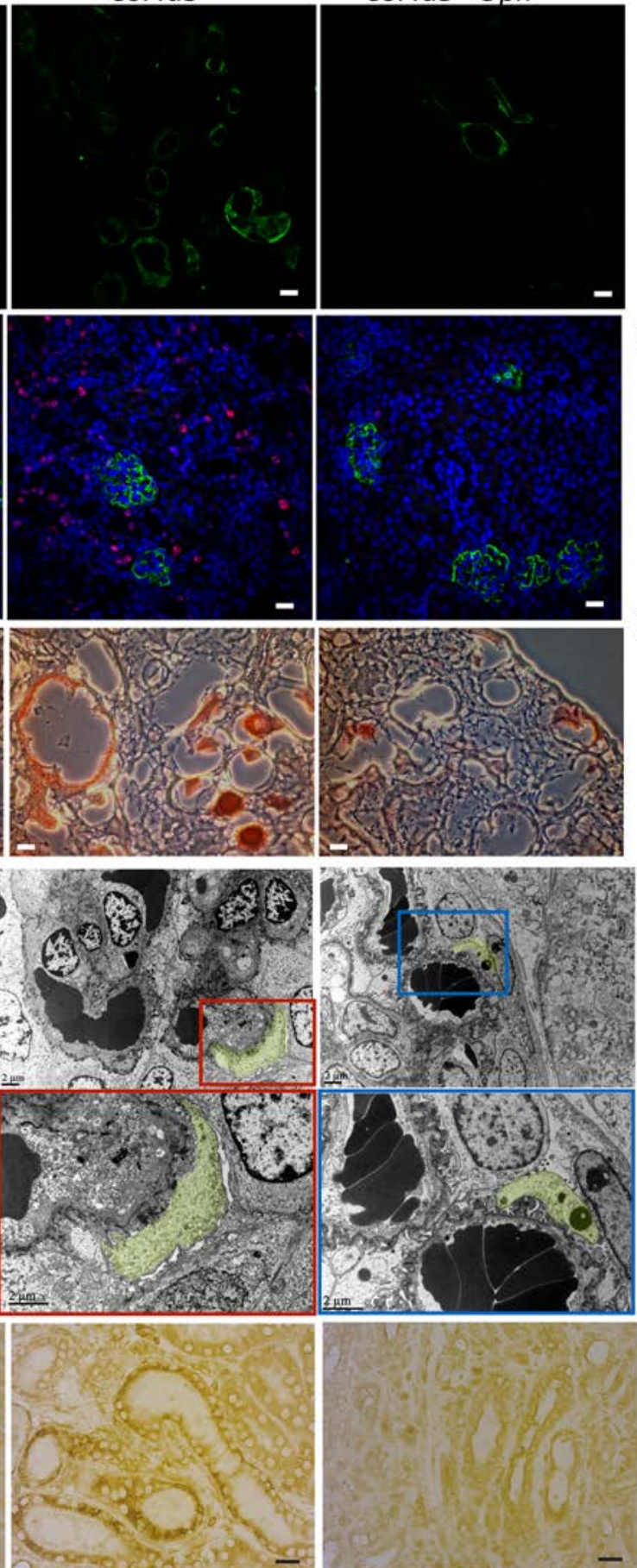

$\mathrm{Col}_{4 a 3} \% \mathrm{Opn}^{\%}$
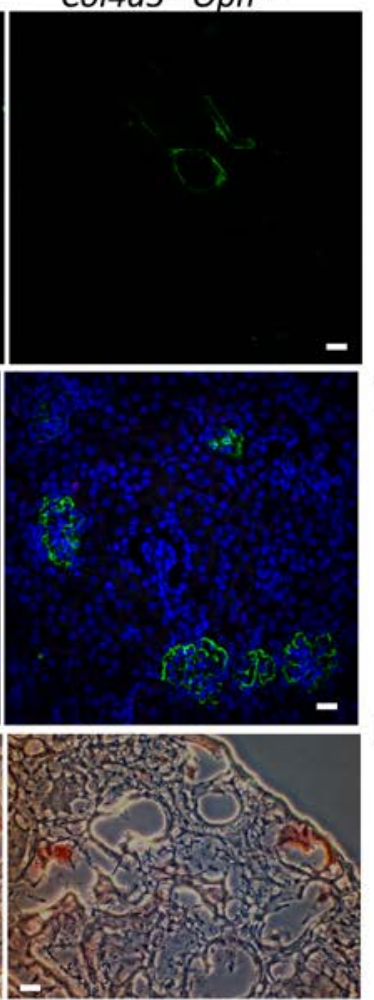

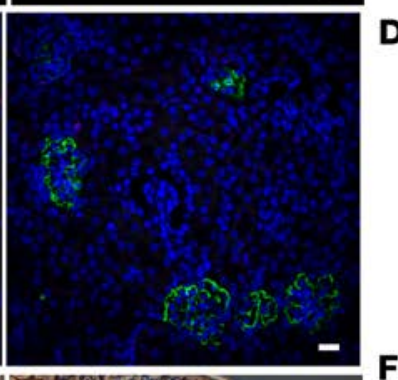

$\mathbf{H}$
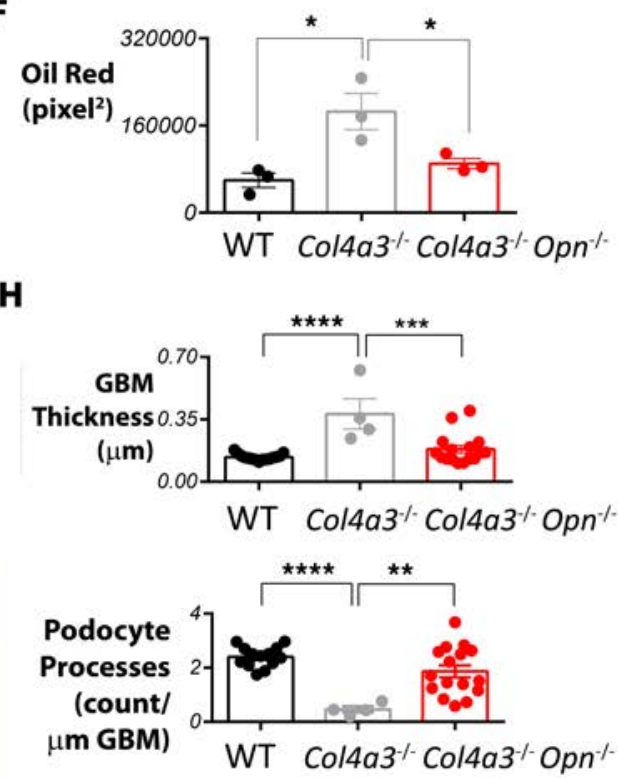

$\mathbf{J}$

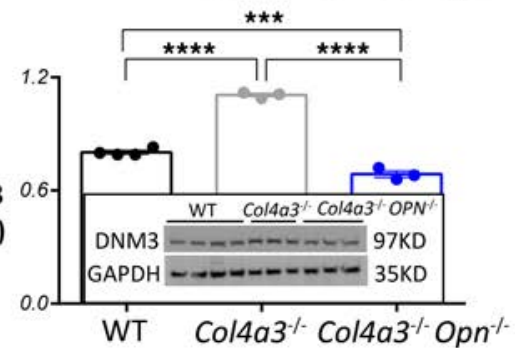

Figure 5. OPN deficiency reduces tubular cell proliferation and prevents severe renal pathology in Alport mouse kidneys. (A and B) Kidney injury molecule-1 (KIM-1) expression is elevated in renal tubules of Alport mice and reduced in Alport osteopontin-deficient (OPN-deficient) mice as shown by immunofluorescence

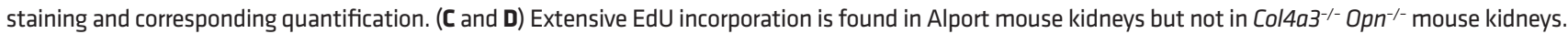
DAPI/nuclear staining is shown in blue. Synaptopodin (SYNPO) staining marks glomeruli. EdU staining is quantified outside the glomeruli. (E and F) Oil Red stain-

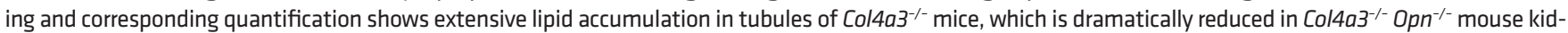
neys. (C) Representative electron microscopy (EM) images show podocyte effacements and thickening of glomerular basement membrane (CBM) in Col4a ${ }^{-/-}$mice

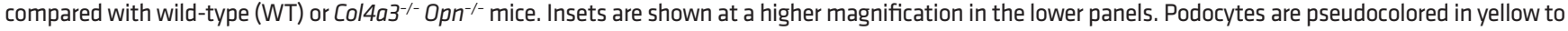
demark the boundary of the GBM. (H) EM images were used to quantify podocyte processes and GBM thickness. $n=4-16$ images were analyzed for quantification of GBM and podocyte effacement. (I) Immunostaining and Western blot (J) show an increased dynamin-3 (DNM3) protein expression in Alport kidneys that

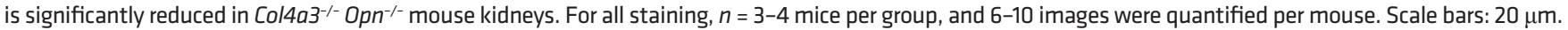
Data are the mean \pm SEM. ${ }^{*} P<0.05 ;{ }^{* *} P<0.01,{ }^{* *} P<0.001,{ }^{* * *} P<0.0001$ based on ANOVA with Tukey's post hoc test. 
A

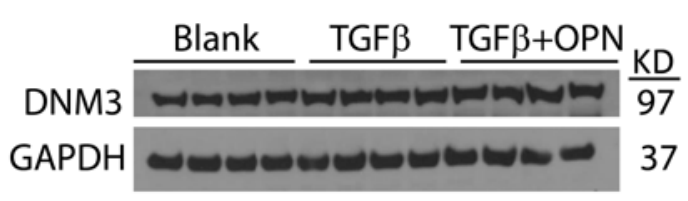

D

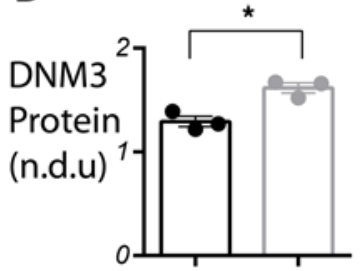

HK2+: SCR DNM3

DNM3 - -

GAPDH ーーーーーー 37

$\mathbf{E}$

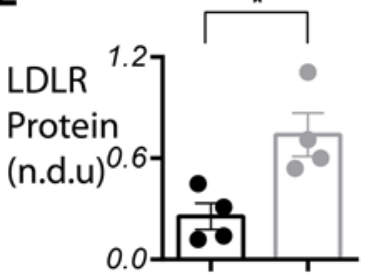

LDLR

GAPDH
B

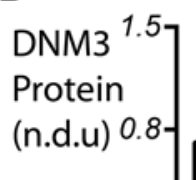

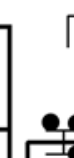

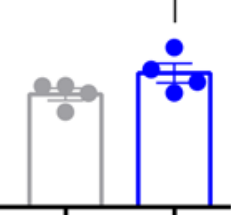

Blank TGF $\beta$ TGF $\beta+O P N$

$\mathbf{F}$

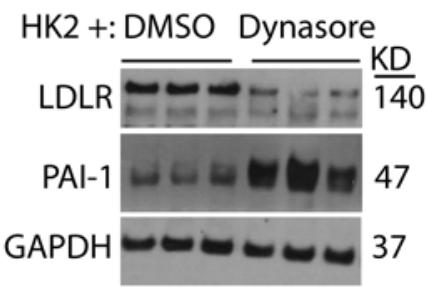

C

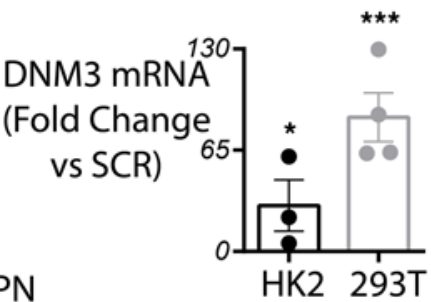

G

WT Primary TECs

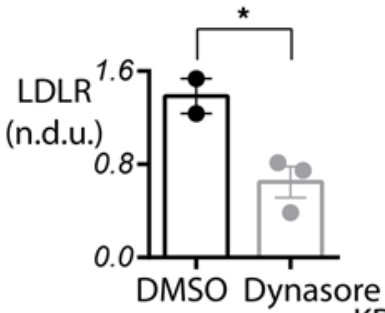

LDLR $=\frac{\mathrm{KD}}{160}$ GAPDH 새 $=37$
Alport Primary TECs

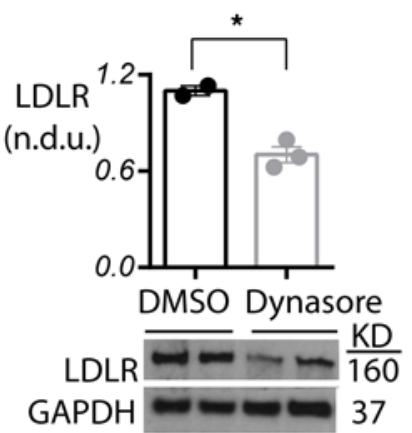

H
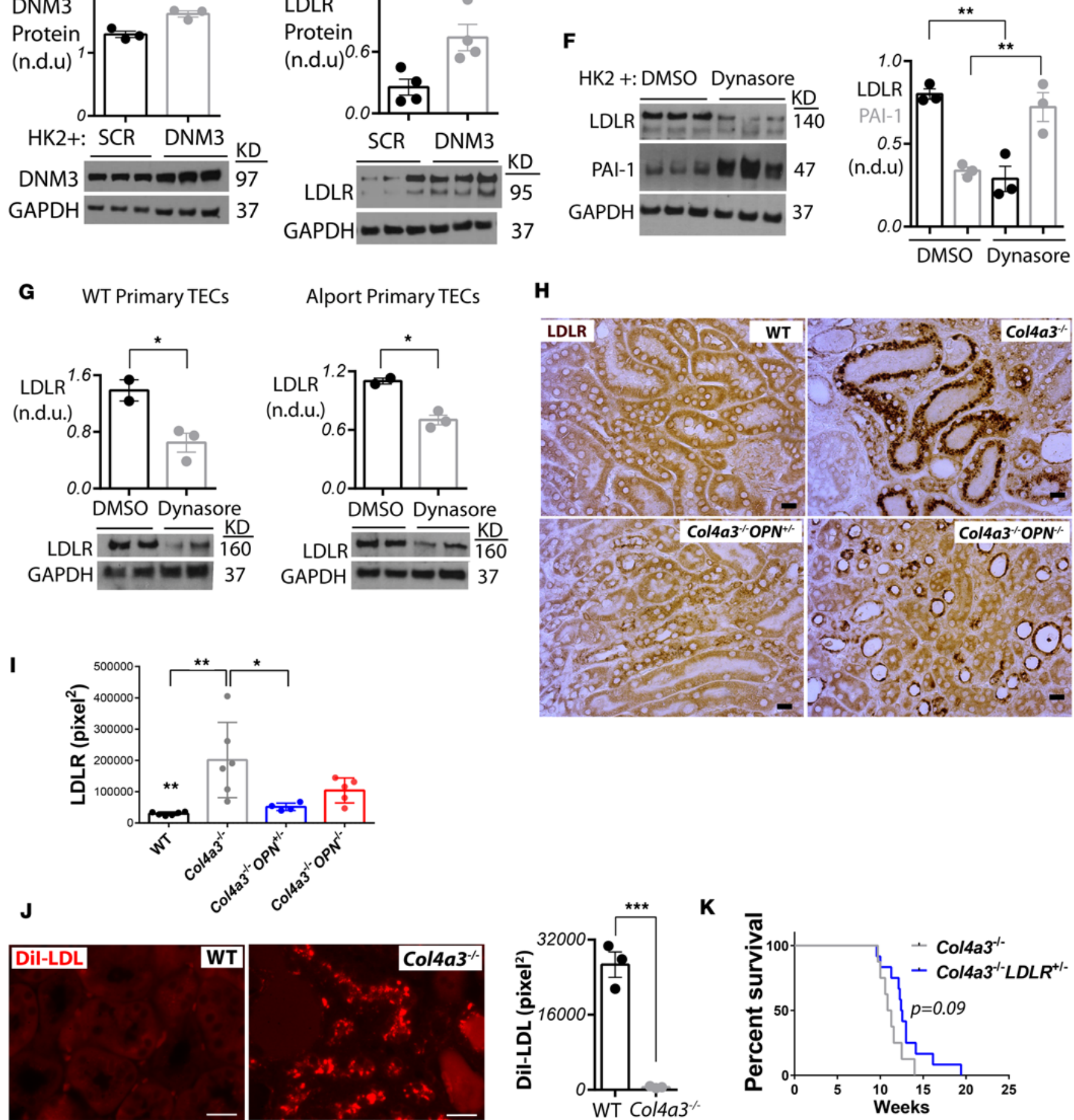
Figure 6. OPN deficiency reduces DNM3-mediated cholesterol influx. (A and B) Osteopontin (OPN) recombinant protein treatment causes increase of dynamin-3 (DNM3) protein expression in HK-2 cells. In panel B, $n=3-4$ per group. Statistics based on ANOVA with Tukey's post hoc test. (C and D) DNM3 was overexpressed by lentivirus in 293T and HK-2 cells and validated by qPCR. SCR, scrambled lentivirus. (E) DNM3 overexpression in HK-2 cells causes overexpression of LDL receptor (LDLR) protein as shown by Western blot. (F) Inhibition of dynamins by dynasore treatment in 30-minute TCF- $\beta$-stimulated HK-2 cells causes upregulation of PAI-1 and downregulation of LDLR protein levels 24 hours after treatment. (G) Inhibition of dynamins by dynasore treatment in 30-minute TGF- $\beta$-stimulated primary tubular epithelial cells (TECs) isolated from wild-type (WT) or Alport mice causes a decrease in LDLR protein levels 24 hours after treatment. In panels C-C, $n=2-4$ per group. Statistics based on Student's $t$ test. n.d.u., normalized densitometry units. (H

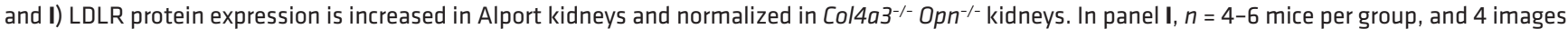
were quantified per mouse. Statistics based on ANOVA with Tukey's post hoc test. Scale bars: $20 \mu \mathrm{m}$. (J) Infusion of $150 \mu \mathrm{g}$ Dil-LDL cholesterol in Alport mice shows a significant 45.9-fold increase in LDL cholesterol uptake by Alport renal tubules after 2 hours. In panel J, $n=3$ mice per group, and 3 images were quantified per mouse. Statistics based on Student's $t$ test. Scale bars: $20 \mu \mathrm{m}$. (K) Heterozygous deletion of LDLR in Alport mice tends to improve survival as shown by Kaplan-Meier curve. $n=8{\mathrm{Co} / 4 a 3^{-/-}}$mice and $n=12 \mathrm{Col}_{4} a 3^{-/-}$Ldlr ${ }^{-1-}$ mice. All mice are littermates. For all, data are the mean $\pm \mathrm{SEM}$. ${ }^{*} P$ $<0.05,{ }^{* *} P<0.01,{ }^{* *} P<0.001,{ }^{* * *} P<0.0001$.

$L d l r^{+-}$mice. We found that disruption of LDL influx by genetic heterozygous deletion of LDLR slightly ( $P$ $=0.09$ ) improved lifespan of Alport mice (Figure $6 \mathrm{~K}$ ). These data suggest a potentially novel pathological pathway in Alport renal tubules in which OPN induces DNM3 expression, which causes increased expression of LDLR and hence elevated cholesterol influx.

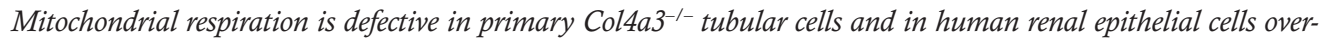
expressing DNM3. Defective fatty acid oxidation (FAO) has been implicated in the development of renal fibrosis in chronic kidney disease (48). To investigate bioenergetics in Alport mouse kidneys, we studied mitochondrial respiration by extracellular flux analysis of oxygen consumption rate (OCR) in primary tubular cells isolated from wild-type and $\mathrm{Col}_{43 a^{-/-}}$mice. Mitochondrial FAO and aerobic glucose oxidation (49) were assessed separately in media supplemented with fatty acids or glucose substrates, respectively. In both FAO and aerobic glucose oxidation assays, basal respiration and spare respiration capacities were significantly lower in the Col43a- tubular cells as compared with wild-type controls (Figure 7, A and B). We also found decreased levels of ATP production in response to oligomycin, maximal respiration levels in response to FCCP (carbonyl cyanide 4-[trifluoromethoxy]phenylhydrazone), and non-mitochondrial respiration levels in response to a mixture of rotenone and antimycin A in Alport tubular cells when compared with wild-type cells (Figure 7, A and B). To test if the defective mitochondrial respiration in Alport kidneys was caused by elevated renal DNM3 expression, we measured OCR in human HK-2 cells stably overexpressing DNM3 lentivirus versus SCR lentivirus. A decreased level of basal respiration was found in HK-2 cells overexpressing DNM3 in both fatty acid- and glucose-based respiration assays (Figure 7, C and D). Additionally, maximal respiration and ATP production were decreased in HK-2 cells overexpressing DNM3 in the FAO assay but not in the glucose-based respiration assay (Figure 7, C and D). Next, we assessed respiration in mitochondria isolated from 2 human kidney cell lines, 293T and HK-2 cells overexpressing DNM3, to dissect which components of the electron transport chain were targeted by DNM3 to regulate mitochondrial bioenergetics in the renal cells. Our results showed that while DNM3 overexpression did not affect basal respiration (state 2) in 293T+DNM3 mitochondria, it did impair basal respiration in HK-2+DNM3 mitochondria. Impaired phosphorylating respiration (state 3), resting respiration (state 4 ), and maximal uncoupling respiration (state $3_{\mathrm{u}}$ ) were found in DNM3-overexpressing 293T and HK-2 mitochondria relative to the SCR/control groups (Figure 7, E and F), all supporting an overall suppressed respiratory function inflicted by DNM3 overexpression. Consistent with defective mitochondrial respiration observed in Col43a ${ }^{-/-}$tubular cells, our EM images revealed stressed mitochondrial morphology in the Alport tubular cells as shown by mitochondrial rupture, mitochondria with disorganized cristae and vesicles, and mitochondria within autophagosomes (Figure 7G). These data unequivocally confirm that mitochondrial respiration is impaired in the Col43a $a^{-/}$mouse kidney and that increased levels of DNM3 (which is regulated by OPN) may play a causative role in mitochondrial dysfunction.

\section{Discussion}

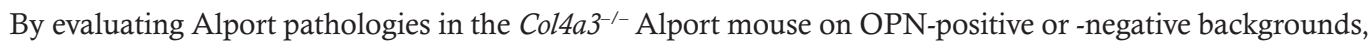
we present here the first-time evidence to the best of our knowledge that OPN is a potential novel therapeutic target for Alport syndrome. Alport patients progress to ESRD at different rates as determined by their underlying mutations and putative modifier genes (50). Like human patients, Col4a $3^{-1-}$ mice on different

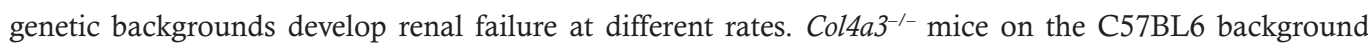
show extended renal survival over the 129 /SvJ background due to the presence of $\alpha 5 / \alpha 6$ (IV) in GBM, 
A

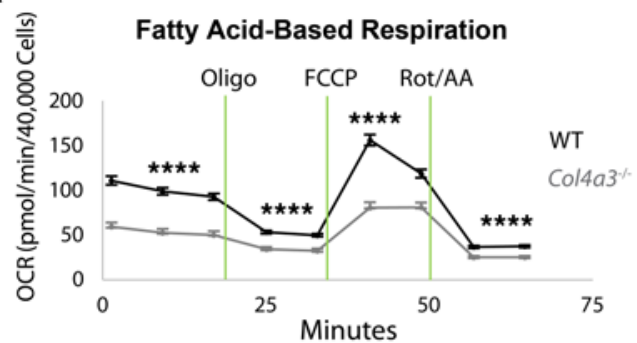

Primary Tubular Epithelial Cells

B

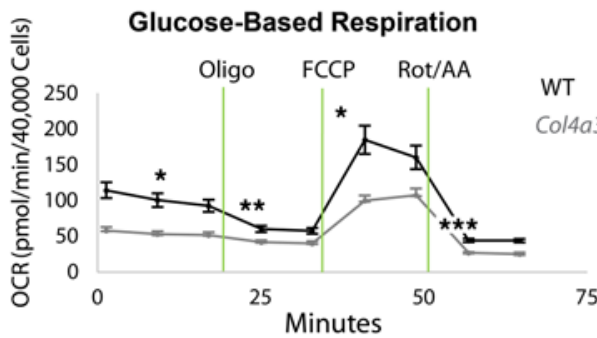

C

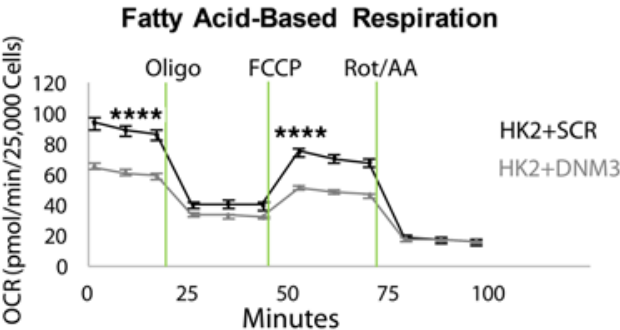

HK2 Cells

HK2 Cells

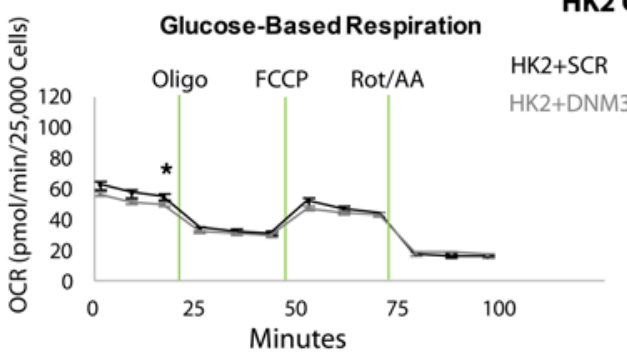

$\mathbf{E}$

293T Mitochondria

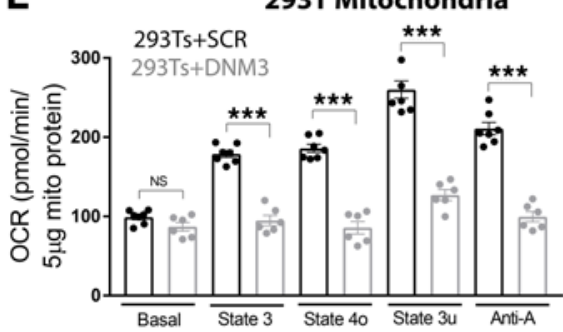

G

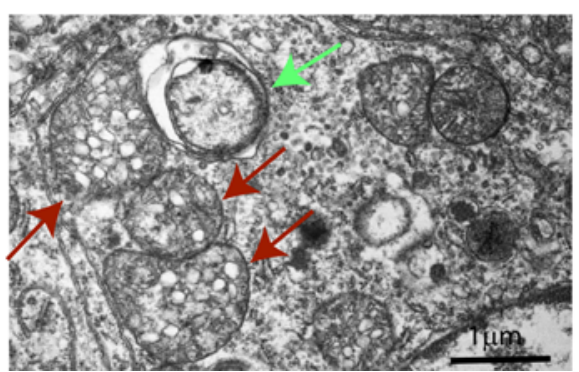

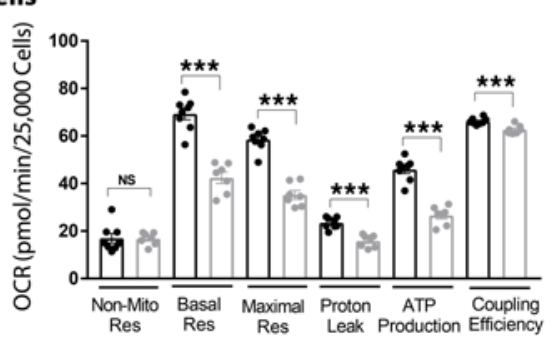
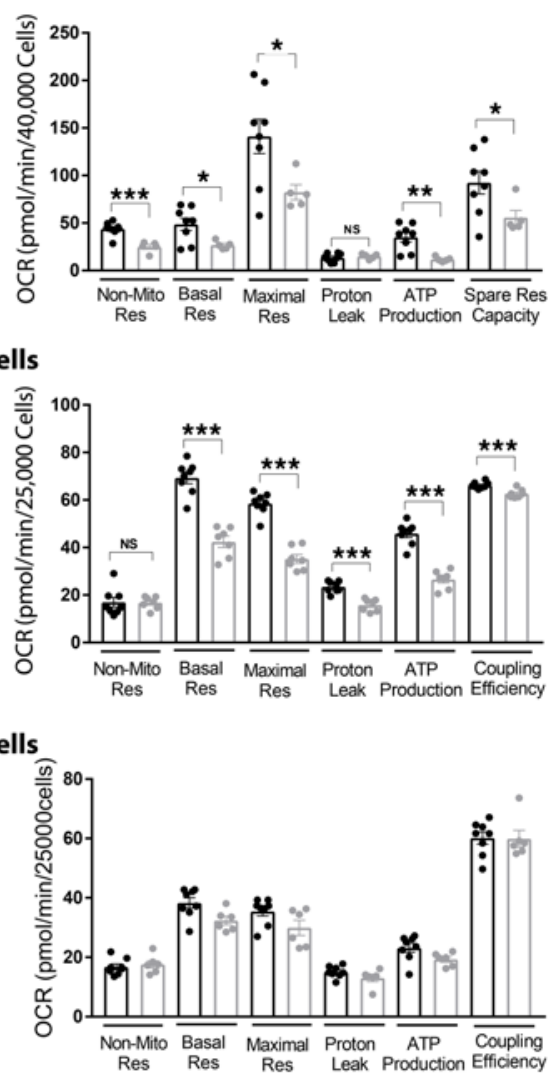

$\mathbf{F}$
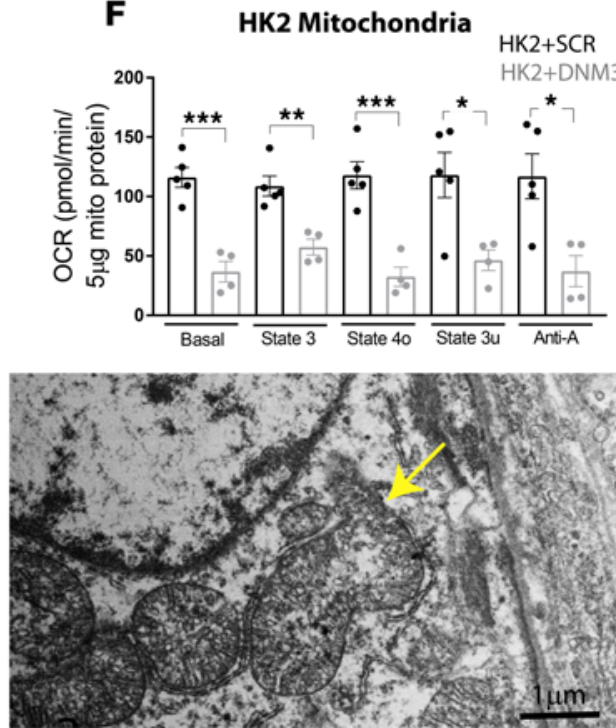

Figure 7. Seahorse assay reveals defective mitochondrial respiration in DNM3-overexpressing cells and in Alport tubular epithelial cells. Mitochondrial respiration capacity is reduced in intact primary tubular epithelial cells isolated from 8-week-old Alport relative to wild-type (WT) kidneys (A and B), or intact HK-2 cells overexpressing dynamin-3 (DNM3) relative to scrambled (SCR) control (C and $\mathbf{D})$. The responses were to $1 \mu \mathrm{M}$ oligomycin (port A), $1 \mu \mathrm{M}$ FCCP (port B), or $2 \mu \mathrm{M}$ rotenone/antimycin $A$ (Rot/AA) (port D) in ( $\mathbf{A}$ and $\mathbf{C}$ ) fatty acid- or (B and D) glucose-based media. Corresponding quantification is shown in the right panels. $n=5-16$ wells per group. Data are the mean $\pm \mathrm{SEM}$. ${ }^{*} P<0.05$, ${ }^{* *} P<0.01$, ${ }^{* * *} P<0.001$, ${ }^{* * * *} P<0.0001$ based on Student's $t$ test. DNM3 overexpression in 293T cells (E) or HK-2 cells (F) causes defective respiration in isolated mitochondria in response to $4 \mathrm{mM} \mathrm{ADP}$ (port A), 2 $\mu \mathrm{M}$ oligomycin (port B), $4 \mu \mathrm{M}$ FCCP (carbonyl cyanide 4-[trifluoromethoxy] phenylhydrazone) (port C), or $10 \mu \mathrm{M}$ antimycin $A$ (port D). Shown are energy states based on quantification of data. $n=6-7$ wells per group of 293T cells and $n=2-3$ wells per group of HK-2 cells. Data are the mean \pm SEM. ${ }^{*} P<$ $0.05,{ }^{* *} P<0.01$ based on Student's $t$ test. NS, not significant. (C) Electron microscopy imaging reveals stressed mitochondria in Alport mouse kidney as shown by mitochondria in autophagosome (green arrow), mitochondria with disrupted cristae morphology (red arrow), and ruptured mitochondria (yellow arrow). Scale bars: $1 \mu \mathrm{m}$. OCR, oxygen consumption rate. 


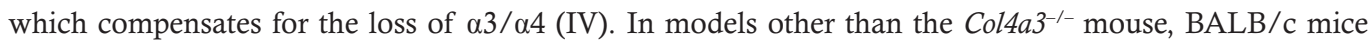
have been shown to be more susceptible to adriamycin (ADR) and develop severe proteinuria, while C57/ B6 mice are resistant to ADR-induced renal pathology (51). In order to mimic the diversity of the human population, all mice used in our study were littermates of multistrain background with roughly similar contribution of 129/SvJ, C57BL6, and BALB/c strains. Although increased OPN has been found in an albuminuria animal model (13) and has controversial roles in the kidney (52), OPN has not been implicated in Alport syndrome. Therefore, our study is the first to our knowledge to report that OPN is significantly overexpressed in the renal tubules and plasma of Alport mice and that OPN has a major causative role in Alport pathology by driving metabolic pathways.

The Alport mouse on the 129/SvJ background suffers from severe proteinuria and ESRD with an average lifespan of 10 weeks (7), which is similar to the lifespan of the Alport mouse on the triple strain that we used. Our study shows an extension of the lifespan up to 22 weeks in OPN-deficient Alport mice. The improved survival in OPN-deficient mice is due to an improved metabolic condition and renal function as reflected by a significant increase in the body weight and decrease in albuminuria in OPN-deficient Alport mice. While plasma BUN and CRE levels can also be used to assess improvement in kidney function in Alport mice $(9,53)$, we observed only a mild and not significant decrease of plasma BUN and CRE in OPN-deficient mice. This may be due to persistent renal inflammation in $\mathrm{CollA3}^{-1-} \mathrm{Opn}^{-/-}$mice shown by CD68 staining. Interestingly, OPN deficiency also increased MCHC. This is clinically relevant because

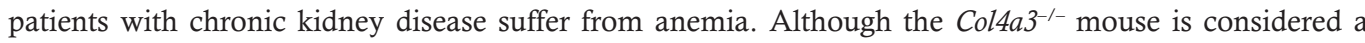
nonhypertensive model of renal fibrosis with reported mean arterial blood pressures (MAPs) close to those of the control groups (54-56), our blood pressure measurements show a mild but significant increase in

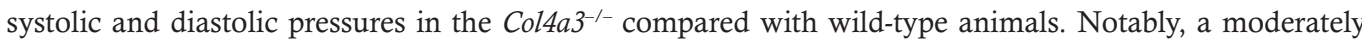
high though nonsignificant MAP of $122 \pm 22 \mathrm{mmHg}$ (versus $107 \pm 12 \mathrm{mmHg}$ in controls, $n=5$ per group) was reported by Gross et al. (56) for $\mathrm{Col}_{43^{-/-}}$mice, which is consistent with our measurements: $126.4 \pm 4.5$ $\mathrm{mmHg}$ (vs. $93.46 \pm 3.53 \mathrm{mmHg}$ in controls, $n=11$ per group, $P=0.0000016$ ). This discrepancy could be due to different mouse strains used (129/SvJ in the previously published studies versus mixed background in our study), or different sample sizes.

Hearing loss is among the early symptoms in Alport patients, where it becomes evident at later childhood or early adolescence (57-59). Disruption of the basement membrane of cochlear stria vascularis associated with hearing deficit has been reported with different pathologies in human Alport patients versus Alport mice. For example, in the $\mathrm{Col}_{4} 3^{-1-}$ Alport mouse model, cochlear pathology is characterized by swollen endothelium and dramatically thickened capillary basement membrane surrounding the stria vascularis (60). This thickened basement membrane phenotype in the Alport mouse confirmed what Wiedauer and Arnold reported for thickened capillary basement membrane in cochleas of Alport patients (61). However, more recent human Alport studies have revealed a normal stria vascularis structure but the presence of a zone of separation between basilar membrane and overlying cells of the organ of Corti, as well as partially torn Reissner's membrane (19-22). Here we show by EM that OPN deletion ameliorates the thickening of the strial capillary basement membrane of Alport mice, suggesting that OPN deficiency

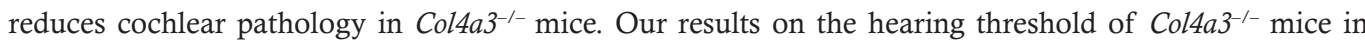
response to both pure tone or click stimuli show that Alport mice on triple genetic background present compromised hearing ability (as is the case with the 129/SvJ strain) and OPN heterozygous deletion tends to ameliorate the hearing deficit. Pharmacological inhibition of OPN in Col4a3 ${ }^{-1-}$ mice rather than genetic deletion is needed to determine the effects of OPN blockade on hearing in the Alport mice without the bias of genetic background, especially given that C57BL6 mice develop hearing deficits earlier in life $(25,26)$. Our potentially novel finding on the role of OPN in hearing function supports the result of a microarray screen from the inner ears of developing PTEN-knockout mice, which suggested a role for OPN signaling in neuronal differentiation of developing auditory neurons (62).

Infrequent yet existing corneal features in Alport patients such as posterior polymorphous dystrophy (63), corneal clouding, and erosion (64) have been associated with altered type IV collagen $\alpha$ chain expression in basement membranes. On the other hand, pathologies of lens capsule are more often found in Alport patients $(65,66)$ and the demonstration of lenticonus is prognostic of Alport syndrome. However, these ocular features have not yet been characterized in the Alport mouse. Anterior lenticonus is more prevalent in male patients with X-linked Alport syndrome, but is common in both men and women with autosomal recessive inheritance. To our knowledge, here we present the first evidence of anterior lenticonus in both male and 
female $\mathrm{Col}_{4} 3^{-/-}$mice similar to Alport patients (2), and show that OPN deficiency reduces lenticonus in $\mathrm{Col}_{4} 3^{-1-}$ mice. Consistent with rare corneal cases reported in human Alport patients, our study shows no obvious corneal pathologies, as CCT and ACD remain unaltered in the Alport mice. A disrupted $\alpha 3 \alpha 4 \alpha 5$ network in the retinal inner limiting membrane and Bruch's membrane cause central or perimacular fleck retinopathy in Alport patients, with peripheral fleck retinopathy more commonly occurring in autosomal recessive disease (27). Interestingly, our EM images reveal significantly thickened membranes around the retinal capillaries that are rescued by OPN deficiency. Whether the thickened basement membranes in the retinal capillaries of $\mathrm{Col}_{4 \mathrm{a}^{-/-}}$mice contribute to the peripheral fleck retinopathy or not calls for future investigations.

We show that KIM-1 expression is elevated in the renal tubules of Alport mice as reported previously (29), and significantly normalized in $\mathrm{Col}_{43^{-1-}} \mathrm{Opn}^{-/-}$mice, indicating the protective impact of OPN deficiency in tubular injury. In addition, increased EdU incorporation suggested a dramatic increase in interstitial cell proliferation in Alport kidneys, which was markedly reduced in OPN-deficient Alport mice. Our EdU data support the antifibrotic role of OPN deletion in Alport kidneys. Moreover, apoptotic cells were reduced in the OPN-deficient Alport kidneys, although OPN deficiency did not appear to reduce inflammation, suggesting involvement of pathways other than antiinflammation in these protective effects observed for the lack of OPN. GBM thickness was also ameliorated in the OPN-deficient Alport kidneys. In addition, we report extensive lipid accumulation in the renal tubules of Alport mice. The lipid accumulation was significantly reduced in OPN-deficient Alport mice. This is in line with a recent report that OPN deficiency also reduced lipid accumulation in kidneys of atherogenic $A p o E^{-/-}$mice (67), presenting OPN as a regulator of lipid homeostasis. These data provide a new insight into the mechanism of OPN function in renal diseases, which is different from its more well-known roles in regulating cell motility and inflammation (68-70) and activation of NF- $\mathrm{kB}$ in albuminuric animals (13). Importantly, our findings emphasize a pivotal role for the tubules in renal injury in Alport syndrome. In fact, tubular epithelial injury is known to contribute to interstitial fibrosis (71), and tubular lipid accumulation has already been implicated in the development of renal fibrosis in chronic kidney disease (48). Although accumulated lipids and foam cells have been implicated in the glomeruli in Alport patients (37), our data on tubular lipid accumulation

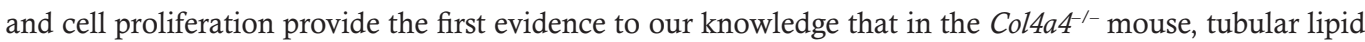
homeostasis is strongly associated with Alport pathology. Because OPN is a matricellular protein that is highly expressed in the tubules, it is logical to think that OPN affects the glomerular structure and function via paracrine and extracellular matrix effects.

Dynamins including DNM3 have been shown to pinch and release clathrin-coated pits from plasma membrane, assisting LDLR-mediated cholesterol endocytosis (72, 73). In addition to cholesterol influx, dynamins have also been implicated in intracellular transportation of cholesterol by delivery of free cholesterol from the early endosome to the endoplasmic reticulum (ER) (73). Inactivation of dynamins by dynasore, a noncompetitive antagonist, retards cellular cholesterol uptake and Niemann-Pick type C-like phenotype (NPC) with the accumulation of swollen late endosomes/lysosomes (LE/LS) (72). However, the exact role of DNM3 and its direct effects on cholesterol uptake or trafficking in renal dysfunction have not been studied. Our data showing OPN regulation of DNM3 and LDLR in vivo in Alport kidneys and in vitro in HK-2 cells, and DNM3 regulation of LDLR in vitro in HK-2 cells and mouse primary tubular epithelial cells suggest that OPN regulates DNM3, which in turn regulates LDLR and subsequent LDL influx. This was validated by a remarkable 45-fold increase in DiI-LDL influx in Alport kidneys, and a modest improvement in survival of the Alport mouse when crossed with $L d l r$-knockout mice that have deficient LDL influx. The modest improvement in survival of Alport mice on the $L d l r$-knockout background could be due to overall increased hypercholesterolemia. It would be interesting to test kidney-specific deletion of LDLR in the Alport mice.

As a highly energetic organ, the kidney is second only to the brain in oxygen consumption primarily through mitochondrial oxidative phosphorylation (74). A recent study has revealed that the development of renal fibrosis is associated with defective mitochondrial FAO in chronic kidney disease (48). However, mitochondrial function has not been investigated in Alport syndrome. Here we are the first to our knowledge to report defective mitochondrial respiration in primary tubular cells isolated from $\mathrm{Col}_{4} 3^{-1-}$ mice as measured by oxygen flux analysis, and disrupted mitochondrial morphology in $\mathrm{Col}_{4} \mathrm{a}^{-1-}$ mouse renal tubules. Although fatty acids are the leading substrate, pyruvate, which is produced by glycolysis in the cytosol, enters mitochondria for complete oxidation in the Krebs cycle (49). Our mitochondrial bioenergetics analysis of primary tubular cells reveals impaired mitochondrial respiratory capacity in both FAO and glucose-based oxidation. Consistent with a previous study that reported DNM1 mutations causing 
mitochondrial dysfunction and cardiomyopathy (75), our findings of increased renal DNM3 suggested a causative role of DNM3 in mitochondrial dysfunction in Col4a $3^{-1-}$ mouse kidneys. Our mechanistic studies using human HK-2 cells overexpressing DNM3 supported impaired mitochondrial respiration. We also take advantage of using isolated mitochondria over intact cells to rule out possible compensatory effects and feedback loops from other organelles, allowing us to directly analyze the effect of DNM3 on mitochondrial respiration (76). Consistently, mitochondria isolated from 293T and HK-2 cells overexpressing DNM3 show defective respiration in state 3 , state $4_{\mathrm{o}}$ and state $3_{\mathrm{u}}$. These results suggest that elevated DNM3 proteins may act through regulating mitochondrial membrane complexes and cause mitochondrial dysfunction in Alport kidneys. Collectively, our findings on the link between DNM3 and defective mitochondrial respiration in Alport syndrome are evidence that DNM3 plays a regulatory role in mitochondria function.

We acknowledge that Alport mice on a B6 background are more resilient than those on a $129 / \mathrm{SvJ}$ background, and our use of mice on a triple strain background may introduce variability on final strain contribution, and hence severity of Alport phenotype. Therefore, we acknowledge this potential limitation in our study. However, the triple strain background may be more clinically relevant provided only littermates are used, as was the case in all of our studies.

In conclusion, we found that OPN deficiency in the Alport mouse rescues capillary basement membrane morphology in kidneys, inner ears, and eyes, and improves renal function, lens structure, and hearing ability. Our data suggest that the rescued renal pathologies are due to reduced DNM3-mediated cholesterol influx and mitochondrial respiration in the renal tubules. Some of our findings such as improved life-span, kidney function, albuminuria, and glomerular membrane thickness were also seen in the antimir-21 treatment study by Gomez et al. (9). It would be interesting to study in the future the therapeutic effect of a combined treatment of Alport mice with antimiR-21 and a blocking OPN agent such as an antibody or aptamer. Our findings also reveal potentially novel regulatory roles of DNM3 in LDLR expression, LDL-cholesterol influx, and mitochondrial respiration, providing evidence for future investigations of developing DNM3 as a therapeutic target in dysregulated cholesterol-associated metabolic syndromes and/or mitochondrial kidney diseases.

\section{Methods}

Animals. Col4a $3^{-1-}$ Alport mice on 129X1/SvJ background were purchased from The Jackson Laboratory and interbred with C57BL/6 $\left(\mathrm{Opn}^{-1-}\right.$ or $\left.\mathrm{Ldl}^{--}\right)$and BALB/c (all from The Jackson Laboratory) mice at least 10 times to generate a mixed line that is approximately one-third of each of the strains. Our breeders' strain was confirmed by a genomic scan in The Jackson Laboratory. In all experiments, mice used were 8-10 weeks of age.

Primary tubular cell isolation and characterization. Wild-type or Alport mice at 8-9 weeks of age were anesthetized under isoflurane flow and perfused with $10 \mathrm{ml}$ PBS followed by $10 \mathrm{ml}$ collagenase type II through the heart apex. Kidneys were minced and incubated in collagenase buffer for 5 minutes at $37^{\circ} \mathrm{C}$ with rotation. Cells were passed through a 70- $\mu \mathrm{m}$ cell strainer, centrifuged at $50 \mathrm{~g}$ for 5 minutes, resuspended in renal epithelial cell media (C-26130, PromoCell) and plated onto 60-mm dishes precoated with collagen (354236, Corning). Homogeneous populations of tubular cells were characterized by staining of angiotensinogen (AGT) according to Kobori et al. (77) as follows: cells were washed with PBS, fixed with 4\% paraformaldehyde (PFA) for 10 minutes, permeabilized with $0.2 \%$ Triton for 3 minutes and blocked with 10\% donkey serum. AGT (23972-1-P, Proteintech) was added to the cells at 1:200 dilution and incubated at $4^{\circ} \mathrm{C}$ overnight. Staining was detected using anti-rabbit Alexa Fluor-488 for 45 minutes. Fluorescence microscopy was used to confirm AGT expression as shown by Supplemental Figure 6.

EdU injections. EdU (A10044, Life Technologies) was prepared as a $1 \mathrm{mg} / \mathrm{ml}$ stock and filtered through $0.2-\mu \mathrm{m}$ Nalgene syringe filter. EdU stock was administered $(100 \mu \mathrm{g})$ daily to each mouse through intraperitoneal injections for up to 30 days to identify mitotic cells. EdU incorporation was visualized by EdU fluorescence staining on paraffin kidney sections.

Injections. DiI-LDL cholesterol (L3482, Thermo Fisher Scientific) was injected (150 $\mu 1$ [150 $\mu \mathrm{g}])$ into

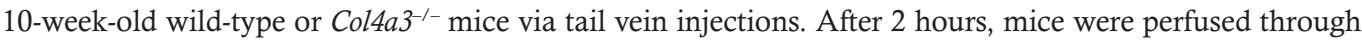
cardiac puncture with $10 \mathrm{ml}$ PBS followed by $10 \mathrm{ml}$ 4\% PFA. Kidneys and livers were collected for histological studies on paraffin sections.

Blood pressure recording. Blood pressure measurements were carried out using the CODA mouse tailcuff system (Kent Scientific). Anesthesia was induced with 5\% vaporized isoflurane at $0.81 / \mathrm{min}$ flow rate and maintained with $1 \%$ isoflurane during blood pressure recordings. Heart rate was monitored and body temperature was controlled within $37^{\circ} \mathrm{C}-39^{\circ} \mathrm{C}$. Two tail cuffs - occlusion and VPR - were equipped 
onto the tails. The occlusion tail cuff was inflated and deflated to control blood flow. The VPR tail cuff was incorporated with a specially designed differential pressure transducer that measured the systolic and diastolic blood pressure by determining the blood volume in the tail. Animals were trained 3 times to minimize stress-related blood pressure changes caused by the system prior to recording the final blood pressure values. Twenty systolic and diastolic blood pressure readings were recorded from each mouse and the 15 most consistent readings were used for analysis.

Hearing testing. ABR was performed on anesthetized mice at 8-9 weeks of age. ABR subdermal electrodes were placed on the vertex, both mastoids, and the left hind leg (ground). Using a commercial system (Intelligent Hearing Systems), electroencephalograms (EEGs) were amplified 100,000 times, band-pass filtered between $30 \mathrm{~Hz}$ and 1,500 Hz, and acquired using a sampling frequency of 10,000 samples per second in 102.4-ms-long epochs. Acoustic stimuli using tone bursts at 4, 8, and $16 \mathrm{kHz}$ were delivered with insert ear phones. For each frequency and epoch, 8 amplitude-modulated tone bursts were presented to each ear at an average stimulation rate of 78.13. The raw EEGs were averaged in blocks of 64 epochs to obtain the auditory evoked potential responses. Each epoch lasted $102.4 \mathrm{~ms}$ and contained 8 tone pips modulated by a 3-ms trapezoidal envelope ( $1 \mathrm{~ms}$ rise time, $1 \mathrm{~ms}$ plateau, and $1 \mathrm{~ms}$ falling time). ABR hearing threshold was defined as the minimum intensity required to produce an $\mathrm{ABR}$ response that was identifiable and consistent. Intensity levels of stimuli were tested in 10-dB sound pressure level intervals.

Anterior segment imaging. Anterior-segment images were captured by OCT using an SD-OCT system (Bioptigen). CCT, ACD, and ACAA were determined using ImageJ software (NIH). CCT was defined as the distance from the corneal epithelial surface to the corneal endothelium. ACD was defined as the distance from the corneal endothelium to the anterior surface of the lens capsule. ACAA was defined as the angle formed by the anterior lens protrusion. A schematic of these measures is shown in Figure 4A.

Triglyceride quantification assay. Snap-frozen kidney tissues $(100 \mathrm{mg})$ collected from non-fasted $\mathrm{Col}_{4 a 3^{-1-}}$,

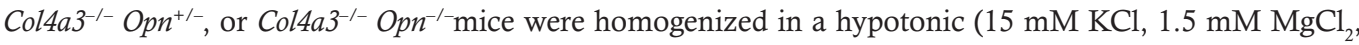
$1 \mathrm{mM}$ DTT, and $10 \mathrm{mM}$ HEPES, pH 7.0) in a Dounce homogenizer. Homogenized solution (200 $\mu \mathrm{l})$ was extracted with heptane/isopropanol $(60 \% \mathrm{v} / \mathrm{v})$ and incubated on ice for 5 minutes. After centrifugation (2,000 $\mathrm{g}$ for 10 minutes), the top organic layer was collected, dried under $\mathrm{N}_{2}$ gas flow, and reconstituted in $100 \mu 1$ isopropanol. Triglycerides were quantified by using $5 \mu \mathrm{l}$ of extracted lipids in a triglyceride quantification kit (Ab65336, Abcam) following the manufacturer's instructions.

ELISA. Albumin concentration was measured by ELISA (E90-134, Bethyl Laboratories) using diluted (1:500-1:20,000) urine samples collected from 8- to 9-week-old mice. CRE concentration was measured using 1:20-diluted urine samples by ELISA (1012, Exocell). Albumin concentrations ( $\mathrm{mg} / \mathrm{ml}$ ) were then normalized to CRE $(\mathrm{mg} / \mathrm{ml})$ for analysis of albuminuria. Plasma galectin-3 levels were measured by ELISA (DY1197, R\&D Systems) according to the manufacturer's instructions.

Blood chemistry. Blood samples were collected into Capiject tubes (T-MLHG, Terumo) by cardiac puncture and centrifuged to separate plasma from blood cells. MCHC, BUN, and CRE levels were analyzed by the Pathology Core Lab at the University of Miami.

Histochemistry. Deparaffinized 4- $\mu \mathrm{m}$ kidney sections were processed for antigen retrieval using citrate buffer in a steamer for 45 minutes, permeabilized using $0.2 \%$ Triton for 15 minutes, and blocked in 10\% donkey serum in 1\% TBST for 1 hour. EdU-555 was incubated for 30 minutes as recommended by the manufacturer. Sections were then counterstained for the glomeruli marker SYNPO (sc-21537, Santa Cruz Biotechnology) at $4^{\circ} \mathrm{C}$ overnight, and staining was detected using anti-goat Alexa Fluor-488 at room temperature for 30 minutes. DNM3 (ab3458, Abcam), DNM2 (ab3457, Abcam), and LDLR (ab52818, Abcam) were stained overnight and detected using biotinylated anti-rabbit (BA-1000, Vector Laboratories) at room temperature for 30 minutes followed by DAB peroxidase (HRP) amplification (SK-4100, Vector Laboratories).

For OPN, KIM-1, or CD68 staining, 10- $\mathrm{mm}$ frozen kidney sections were air dried, rinsed with PBS, permeabilized with $0.2 \%$ Triton for 3 minutes, and blocked with $10 \%$ donkey serum for 30 minutes at room temperature. OPN (AF808, R\&D Systems), Kim-1 (AF3689, R\&D Systems), or CD68 (MCA1957, Bio-Rad) antibodies were used at $4^{\circ} \mathrm{C}$ overnight and detected using anti-goat or anti-rat Alexa Fluor-488 at room temperature for 30 minutes. For immunostaining, primary and secondary antibody incubations were done in $10 \%$ donkey serum in $1 \times$ TBST. For Oil Red staining, frozen sections were processed as described above and incubated with $60 \%$ isopropanol for 5 minutes followed by Oil Red solution for 45 minutes. For filipin staining, frozen kidney sections were fixed in 4\% PFA, washed with PBS, and incubated with 0.25 $\mathrm{mg} / \mathrm{ml}$ filipin working solution for 2 hours at room temperature (78). TUNEL staining was performed on 
frozen kidney sections following the manufacturer's instructions (AB66110, Abcam). DNM3, LDLR, and Oil Red staining images were captured on a Zeiss microscope using a $\times 32$ objective and TUNEL images were captured using a $\times 40$ objective. OPN, KIM-1, CD68, and EdU fluorescent images were captured on a Zeiss LSM710 confocal microscope using Z-scanning at $\times 40$ magnification. Three to 6 kidneys/mice per group were used and 5-10 images per kidney were acquired and quantified. For immunostaining, images were quantified using Fiji ImageJ. For Oil Red staining, 3 kidneys/mice per group were used and 5 images were acquired and quantified using ImageJ. For TUNEL staining, apoptotic cells were counted per field with a $\times 40$ objective and 5 different fields were used for quantification.

EM. Kidneys, cochlea, and eyes were fixed for 24 hours in $10 \%$ formalin, followed by 24 hours in $2 \%$ glutaraldehyde, rinsed in wash buffer 3 times, and then postfixed in $2 \%$ osmium tetroxide in $0.1 \mathrm{M}$ phosphate buffer overnight. After buffer rinses, tissues were dehydrated through a graded series of ethanol and embedded using Embed/Araldite (Electron Microscopy Sciences) overnight in a $64^{\circ} \mathrm{C}$ oven. Silver/gold sections were cut on a Leica Ultracut $\mathrm{R}$ and stained in uranyl acetate and lead citrate. Images were captured by a Gatan Orius SC 200D CCD camera) in a JEM 1400 electron microscope (JEOL).

Quantification of GBM thickness and podocyte processes. The thickness of the GBM and podocyte processes were quantified using the methods described previously (79). Three parameters were measured with the image analysis software, ImageJ (NIH): GBM thickness, GBM length, and podocyte foot process number. The GBM thickness was measured using ImageJ's straight-line tool perpendicular to the membrane. The GBM length was measured using ImageJ's freehand line tool and the number of podocyte foot processes were counted in each GBM with the multi-point tool. For each capillary, the mean number of podocyte foot processes per distance measured was reported.

Lentiviral infections. 293T and HK-2 cells were seeded at $2.5 \times 10^{5}$ cells per dish and infected with either a CMV-scrambled-GFP (LVP015-G, ABM) or a CMV-DNM3-GFP (LVP140689, ABM) lentivirus at MOI = 1 overnight. The next day, infection media were changed to fresh culture media and $5 \mu \mathrm{g} / \mathrm{ml}$ puromycin was supplemented for stable selections. After expansion, the cells were sorted for the top 5\% GFP-positive cells. Selected cells were maintained in $5 \mu \mathrm{g} / \mathrm{ml}$ puromycin-supplemented culture media at all stages during experiments.

Mitochondrial isolation and Seahorse assay. Mitochondrial isolation buffer (MSHE+BSA) was prepared using $70 \mathrm{mM}$ sucrose, $210 \mathrm{mM}$ mannitol, $5 \mathrm{mM}$ HEPES, $1 \mathrm{mM}$ EGTA, and 0.5\% (w/v) fatty acid-free BSA with $\mathrm{pH}$ adjusted to 7.2. Mitochondrial assay solution (MAS, $1 \times$ ) was prepared using $70 \mathrm{mM}$ sucrose, $220 \mathrm{mM}$ mannitol, $10 \mathrm{mM} \mathrm{KH}_{2} \mathrm{PO}_{4}, 5 \mathrm{mM} \mathrm{MgCl}_{2}, 2 \mathrm{mM}$ HEPES, $1 \mathrm{mM}$ EGTA, and $0.2 \%$ (w/v) fatty acid-free BSA and adjusted $\mathrm{pH}$ to 7.2 at $37^{\circ} \mathrm{C}$ with potassium hydroxide. A $2 \times$ stock of MAS was prepared to dilute substrates, ADP, and respiration reagents. Stocks of succinate, malate, pyruvate (500 $\mathrm{mM})$, ADP (100 mM), ascorbate (1 M), $N, N, N^{\prime}, N^{\prime}$-tetramethyl-p-phenylenediamine (TMPD, $10 \mathrm{mM}$ plus $10 \mathrm{mM}$ ascorbate) were made in $\mathrm{H}_{2} \mathrm{O}$ and adjusted to $7.2 \mathrm{pH}$ with potassium hydroxide. FCCP $(50 \mathrm{mM}), 10 \mathrm{mM}$ rotenone, $10 \mathrm{mM}$ oligomycin, and $10 \mathrm{mM}$ antimycin A stocks were made in DMSO. All reagents were stored at $-20^{\circ} \mathrm{C}$, except pyruvate, which was prepared fresh on the day of each experiment.

Mitochondria were isolated from confluent 293T (ATCC CRL-11268) or HK-2 (ATCCCRL-2190) cells cultured in 100-mm plates. Cells were collected in $5 \mathrm{ml}$ of MSHE+BSA on ice and homogenized in a Dounce homogenizer in 5 strokes with a 1-minute interval between each stroke using the tight pestle. The homogenate was centrifuged at $800 \mathrm{~g}$ for 10 minutes at $4^{\circ} \mathrm{C}$ to remove nuclei in the pellet and the collected supernatant was then centrifuged at $10,000 \mathrm{~g}$ for 10 minutes at $4^{\circ} \mathrm{C}$ to pellet mitochondria. The pellet was resuspended in $4 \mathrm{ml}$ of $\mathrm{MSHE}+\mathrm{BSA}$ and centrifuged again at $10,000 \mathrm{~g}$ for 10 minutes at $4^{\circ} \mathrm{C}$ and finally the pellet was resuspended in $100 \mu 1 \times$ MAS as mitochondrial protein solution. The concentration of mitochondrial protein $(\mu \mathrm{g} / \mu \mathrm{l})$ was determined using the Bradford assay (Bio-Rad). Mitochondrial protein $(5 \mu \mathrm{g})$ was added per well to a 96-well Seahorse microplate in a total volume of $50 \mu 1$ in initial media including pyruvate $(10 \mathrm{mM})$, malate $(2 \mathrm{mM})$, and FCCP $(4 \mu \mathrm{M})$ for the coupling assay. The plate was then centrifuged at 2,000 $\mathrm{g}$ for 20 minutes at $4^{\circ} \mathrm{C}$ for mitochondria to attach firmly to the bottom of the assay plate. At the same time, each of the following compounds was loaded into the corresponding ports on a Seahorse cartridge and calibrated in the XF96 instrument: port A, ADP (4 mM); port B, oligomycin $(2 \mu \mathrm{M})$; port C, FCCP $(4 \mu \mathrm{M})$; and port D, antimycin A $(10 \mu \mathrm{M})$. After centrifugation, attached mitochondrial protein received $125 \mu \mathrm{l}$ initial media per well and the plate was warmed up in a $\mathrm{CO}_{2}$-free incubator for 10 minutes and the XF assay was performed using an XF96 Extracellular Flux Analyzer (Seahorse Bioscience).

For primary tubular cells isolated from 8-week-old wild-type or Alport mice, cells were seeded at 40,000 cells per well on XF96 V3-PS cell culture microplates precoated with collagen. For HK-2 cells (stably over- 
expressing DNM3 or SCR lentivirus), cells were seeded at 25,000 cells per well precoated with cell media. The next day (approximately 20 hours after seeding), the seeded cells were analyzed by Seahorse assays in 2 different media for measuring FAO or glucose-based respiration. For FAO assay, media was supplemented with $100 \mu \mathrm{M}$ BSA-palmitate, $200 \mu \mathrm{M}$ L-carnitine, and $10 \mathrm{mM}$ 2-deoxyglucose (to inhibit glycolysis). Glucose medium was supplemented with $17.5 \mathrm{mM}$ glucose, $100 \mu \mathrm{M}$ control BSA (without palmitate), $200 \mu \mathrm{M}$ L-carnitine, and $20 \mu \mathrm{M}$ etomoxir (to inhibit FAO). Mitochondrial function was assessed through measurement of OCR changes using an XF96 analyzer in response to the following compounds: $1 \mu \mathrm{M}$ oligomycin (port A), $1 \mu \mathrm{M}$ FCCP (port B), and $2 \mu \mathrm{M}$ rotenone/antimycin A mixture (port C). OCR data were then normalized to cell counts measured by fluorescence reading at $355 \mathrm{~nm}$ excitation and $460 \mathrm{~nm}$ emission following Hoechst staining (80).

Microarrays and bioinformatics. Kidneys from 2 wild-type, $3 \mathrm{Col}_{4 a 3^{-1-}}$, and $3 \mathrm{Col}_{4} \mathrm{a}^{-/-} \mathrm{Opn}^{-/-}$mice were each lysed using Cell Disruption Buffer (Mirvana Paris Kit) and total RNA was extracted using Mirvana Paris Kit to yield a 260-280 nm absorbance ratio of 2.0. RNA concentration and integrity was determined by using an Agilent Bioanalyzer. A 1- $\mu$ g aliquot of total RNA from each of the samples was processed using instructions and reagents supplied by the manufacturer (Affymetrix). Briefly, total RNA was reverse transcribed using a T7-Oligo (dT) Promoter Primer in the first-strand cDNA synthesis reaction. Following RNase H-DNA polymerase I-mediated second-strand cDNA synthesis, the double-stranded cDNA was purified and served as a template for in vitro transcription in the presence of T7 RNA polymerase and a biotinylated nucleotide analog/ribonucleotide mix, producing biotin-labeled complementary RNA (cRNA). cRNA probes were then purified, fragmented, and hybridized on Mouse ST2 gene expression arrays (45,101 probe sets). Background noise, housekeeping gene expression, and $3^{\prime} / 5^{\prime}$ ratio values of all chips were within quality control limits set by Affymetrix. Expression ratios were calculated as the power- 2 exponential of the log 2 differences. The acceptance criteria for gene array expression changes was a minimum 2-fold change in $\log 2$ (equivalent to 4-fold) and a 1-way ANOVA $t$ test $P$ value of less than 0.05. All microarray raw files were submitted to the NCBI Gene Expression Omnibus (GEO) database (GSE109777).

Western blots. Protein concentration was measured using a Bradford assay. Samples were prepared and separated by a 4\%-12\% Novex mini 15-well gradient gel (Bolt System, Life Technologies), and probed with OPN (AF808, R\&D Systems), DNM3 (ab3458, Abcam), LDLR (ab52818, Abcam), and GAPDH (sc-25778, Santa Cruz Biotechnology) antibodies. Signals were detected by chemiluminescence (Femto, Thomas Scientific) on photographic films. Digitized images were analyzed using ImageJ (NIH). Protein band densitometry was normalized to that of GAPDH, and the averaged results were plotted as normalized densitometry units.

Statistics. For all experiments, $n$ refers to the number of individual mice or individual culture plates. All data are expressed as mean \pm SEM. $P$ values comparing 2 groups were calculated using Student's $t$ tests. For more than 2 groups, $P$ values were calculated using ANOVA and corrected for multiple comparisons using Tukey's post hoc test in GraphPad Prism. Repeated symbols represent $P$ values of different orders of magnitude, i.e., ${ }^{*} P<0.05,{ }^{* *} P<0.01,{ }^{* * *} P<0.001$, and ${ }^{* * * *} P<0.0001$.

Study approval. All experiments involving animals were approved by the IACUC at the University of Miami, conforming to NIH guidelines.

\section{Author contributions}

WD, KY, and LAS designed the study, performed experiments, and analyzed the data. BJG designed the hearing testing studies. SG designed, conducted, and analyzed the hearing testing study. PR and GL performed the dynasore-treatment experiments. WD and ALS designed and conducted the OCT study. ALS analyzed the OCT studies. AJM designed the triglyceride study. AK captured and analyzed EM images. WD and LAS wrote the manuscript.

\section{Acknowledgments}

This work was supported by the following grants to L.A. Shehadeh: NIH (R56HL132209), Miami Heart Research Institute, and the American Heart Association (AHA) Grant-in-Aid (14GRNT19960000). Wen Ding is a recipient of a Sublett AHA predoctoral fellowship (15PRE22450019). We would like to thank Margaret Bates and Vania W. Almeida from the Electron Microscope Core Facility at the University of Miami, Miller School of Medicine, for their services. We thank Maria Bulina from the Imaging Core Facility for her assistance with quantification of staining. We would also like to thank Craig Brooks from the University of Vanderbilt for his help with renal mitochondrial EM analysis. We thank Thomas VanDeWater for the use of ABR equipment and advice. 
Address correspondence to: Lina A. Shehadeh, Interdisciplinary Stem Cell Institute, Biomedical Research Building, Room 818, University of Miami Leonard M. Miller School of Medicine, 1501 NW 10th Avenue, Miami, Florida 33136, USA. Phone: 305.243.0867; Email:1shehadeh@med.miami.edu.

1. Alport AC. Hereditary familial congenital haemorrhagic nephritis. Br Med J. 1927;1(3454):504-506.

2. Mahajan S. PRA, Bairy M., Hasan F., Sahoo P. Alport's syndrome: a study of systemic manifestations. International Journal of Scientific Study. 2013;1(2):4.

3. Grünfeld JP. Contemporary diagnostic approach in Alport's syndrome. Ren Fail. 2000;22(6):759-763.

4. Miner JH, Sanes JR. Collagen IV alpha 3, alpha 4, and alpha 5 chains in rodent basal laminae: sequence, distribution, association with laminins, and developmental switches. J Cell Biol. 1994;127(3):879-891.

5. Abrahamson DR, Hudson BG, Stroganova L, Borza DB, St John PL. Cellular origins of type IV collagen networks in developing glomeruli. J Am Soc Nephrol. 2009;20(7):1471-1479.

6. Kalluri R, Shield CF, Todd P, Hudson BG, Neilson EG. Isoform switching of type IV collagen is developmentally arrested in $\mathrm{X}$-linked Alport syndrome leading to increased susceptibility of renal basement membranes to endoproteolysis. J Clin Invest. 1997;99(10):2470-2478.

7. Cosgrove D, et al. Collagen COL4A3 knockout: a mouse model for autosomal Alport syndrome. Genes Dev. 1996;10(23):2981-2992

8. Cosgrove D, et al. Ultrastructural, physiological, and molecular defects in the inner ear of a gene-knockout mouse model for autosomal Alport syndrome. Hear Res. 1998;121(1-2):84-98.

9. Gomez IG, et al. Anti-microRNA-21 oligonucleotides prevent Alport nephropathy progression by stimulating metabolic pathways. J Clin Invest. 2015;125(1):141-156.

10. Kang JS, et al. Loss of alpha3/alpha4(IV) collagen from the glomerular basement membrane induces a strain-dependent isoform switch to alpha5alpha6(IV) collagen associated with longer renal survival in Col4a3-/- Alport mice. J Am Soc Nephrol. 2006;17(7):1962-1969.

11. Sodek J, Ganss B, McKee MD. Osteopontin. Crit Rev Oral Biol Med. 2000;11(3):279-303.

12. Li J, Yousefi K, Ding W, Singh J, Shehadeh LA. Osteopontin RNA aptamer can prevent and reverse pressure overload-induced heart failure. Cardiovasc Res. 2017;113(6):633-643.

13. Lorenzen J, et al. The role of osteopontin in the development of albuminuria. J Am Soc Nephrol. 2008;19(5):884-890.

14. Dimas G, Iliadis F, Grekas D. Matrix metalloproteinases, atherosclerosis, proteinuria and kidney disease: Linkage-based approaches. Hippokratia. 2013;17(4):292-297.

15. Li SY, et al. Matrix metalloproteinase-9 deficiency attenuates diabetic nephropathy by modulation of podocyte functions and dedifferentiation. Kidney Int. 2014;86(2):358-369.

16. Quigley R. Chronic kidney disease: highlights for the general pediatrician. Int J Pediatr. 2012;2012:943904.

17. Gillion V, Jadoul M, Aydin S, Godefroid N. ANCA vasculitis in a patient with Alport syndrome: a difficult diagnosis but a treatable disease! BMC Nephrol. 2017;18(1):105.

18. Breyer MD, Susztak K. The next generation of therapeutics for chronic kidney disease. Nat Rev Drug Discov. 2016;15(8):568-588.

19. Rudolf Glueckert AJ, Anneliese Sschrott-Fisher, Ciorba A. Inner ear histopathological findings in Alport syndrome. Audiol Med. 2007;5(2):9.

20. Zehnder AF, et al. Distribution of type IV collagen in the cochlea in Alport syndrome. Arch Otolaryngol Head Neck Surg. 2005;131(11):1007-1013.

21. Merchant SN, et al. Temporal bone histopathology in alport syndrome. Laryngoscope. 2004;114(9):1609-1618.

22. Smith RJ, Steel KP, Barkway C, Soucek S, Michaels L. A histologic study of nonmorphogenetic forms of hereditary hearing impairment. Arch Otolaryngol Head Neck Surg. 1992;118(10):1085-1094.

23. Li J, Yousefi K, Ding W, Singh J, Shehadeh LA. Osteopontin RNA aptamer can prevent and reverse pressure overload-induced heart failure. Cardiovasc Res. 2017;113(6):633-643.

24. Cosgrove D, Kalluri R, Miner JH, Segal Y, Borza DB. Choosing a mouse model to study the molecular pathobiology of Alport glomerulonephritis. Kidney Int. 2007;71(7):615-618.

25. Ison JR, Allen PD, O'Neill WE. Age-related hearing loss in C57BL/6J mice has both frequency-specific and non-frequencyspecific components that produce a hyperacusis-like exaggeration of the acoustic startle reflex. J Assoc Res Otolaryngol. 2007;8(4):539-550.

26. Bowl MR, Dawson SJ. The mouse as a model for age-related hearing loss - a mini-review. Gerontology. 2015;61(2):149-157.

27. Savige J, Sheth S, Leys A, Nicholson A, Mack HG, Colville D. Ocular features in Alport syndrome: pathogenesis and clinical significance. Clin J Am Soc Nephrol. 2015;10(4):703-709.

28. Jarrín E, Jarrín I, Arnalich-Montiel F. Lenticonus diagnosis in Alport's syndrome: Anterior capsule apical angle calculation using Scheimpflug imagery. Arch Soc Esp Oftalmol. 2015;90(8):379-381.

29. Jarad G, Knutsen RH, Mecham RP, Miner JH. Albumin contributes to kidney disease progression in Alport syndrome. Am J Physiol Renal Physiol. 2016;311(1):F120-F130.

30. Han WK, Bailly V, Abichandani R, Thadhani R, Bonventre JV. Kidney Injury Molecule-1 (KIM-1): a novel biomarker for human renal proximal tubule injury. Kidney Int. 2002;62(1):237-244.

31. Humphreys BD, et al. Chronic epithelial kidney injury molecule-1 expression causes murine kidney fibrosis. J Clin Invest. 2013;123(9):4023-4035.

32. Worgall TS. Lipid metabolism in cystic fibrosis. Curr Opin Clin Nutr Metab Care. 2009;12(2):105-109.

33. Clemens MR, Einsele H, Remmer H. Alcohol consumption and hepatic fibrosis affect the fatty acid composition of red blood cells and their susceptibility to lipid peroxidation. Arch Toxicol. 1987;60(1-3):167-169.

34. Hattori M, et al. Mechanisms of glomerular macrophage infiltration in lipid-induced renal injury. Kidney Int Suppl. 
1999;71:S47-S50

35. Balson KR, Niall JF, Best JD. Glomerular lipid deposition and proteinuria in a patient with familial dysbetalipoproteinaemia. $J$ Intern Med. 1996;240(3):157-159.

36. Fornoni A, Merscher S, Kopp JB. Lipid biology of the podocyte--new perspectives offer new opportunities. Nat Rev Nephrol. 2014;10(7):379-388.

37. White RH, Raafat F, Milford DV, Komianou F, Moghal NE. The Alport nephropathy: clinicopathological correlations. Pediatr Nephrol. 2005;20(7):897-903.

38. Rittling SR. Osteopontin in macrophage function. Expert Rev Mol Med. 2011;13:e15.

39. Rodgers $\mathrm{KD}$, et al. Monocytes may promote myofibroblast accumulation and apoptosis in Alport renal fibrosis. Kidney Int. 2003;63(4):1338-1355.

40. Dennis J, et al. Collagen XIII induced in vascular endothelium mediates alphalbeta1 integrin-dependent transmigration of monocytes in renal fibrosis. Am J Pathol. 2010;177(5):2527-2540.

41. Whalen RE, Huang S, Peschel E, McIntosh HD. Hereditary nephropathy, deafness and renal foam cells. Am J Med. 1961;31:171-186.

42. Saleh S, Thompson DE, McConkey J, Murray P, Moorehead RA. Osteopontin regulates proliferation, apoptosis, and migration of murine claudin-low mammary tumor cells. BMC Cancer. 2016;16:359.

43. Soda K, et al. Role of dynamin, synaptojanin, and endophilin in podocyte foot processes. J Clin Invest. 2012;122(12):4401-4411. 44. Hahm K, et al. Alphav beta6 integrin regulates renal fibrosis and inflammation in Alport mouse. Am J Pathol. 2007;170(1):110-125

45. Sayers R, Kalluri R, Rodgers KD, Shield CF, Meehan DT, Cosgrove D. Role for transforming growth factor-beta1 in alport renal disease progression. Kidney Int. 1999;56(5):1662-1673.

46. Chung CL, et al. Dynasore, a dynamin inhibitor, induces PAI-1 expression in MeT-5A human pleural mesothelial cells. Am J Respir Cell Mol Biol. 2009;40(6):692-700.

47. Ishibashi S, Brown MS, Goldstein JL, Gerard RD, Hammer RE, Herz J. Hypercholesterolemia in low density lipoprotein receptor knockout mice and its reversal by adenovirus-mediated gene delivery. J Clin Invest. 1993;92(2):883-893.

48. Kang HM, et al. Defective fatty acid oxidation in renal tubular epithelial cells has a key role in kidney fibrosis development. Nat Med. 2015;21(1):37-46.

49. Rich PR. The molecular machinery of Keilin's respiratory chain. Biochem Soc Trans. 2003;31(Pt 6):1095-1105.

50. Kamiyoshi N, et al. Genetic, clinical, and pathologic backgrounds of patients with autosomal dominant Alport syndrome. Clin $J$ Am Soc Nephrol. 2016;11(8):1441-1449.

51. Goldwich A, Steinkasserer A, Gessner A, Amann K. Impairment of podocyte function by diphtheria toxin--a new reversible proteinuria model in mice. Lab Invest. 2012;92(12):1674-1685.

52. Xie Y, Sakatsume M, Nishi S, Narita I, Arakawa M, Gejyo F. Expression, roles, receptors, and regulation of osteopontin in the kidney. Kidney Int. 2001;60(5):1645-1657.

53. Petrova DT, et al. Effects of mycophenolate mofetil on kidney function and phosphorylation status of renal proteins in Alport COL4A3-deficient mice. Proteome Sci. 2014;12(1):56.

54. Gross O, et al. Preemptive ramipril therapy delays renal failure and reduces renal fibrosis in COL4A3-knockout mice with Alport syndrome. Kidney Int. 2003;63(2):438-446.

55. Gross O, Girgert R, Rubel D, Temme J, Theissen S, Müller GA. Renal protective effects of aliskiren beyond its antihypertensive property in a mouse model of progressive fibrosis. Am J Hypertens. 2011;24(3):355-361.

56. Gross $\mathrm{O}$, et al. Antifibrotic, nephroprotective potential of ACE inhibitor vs AT1 antagonist in a murine model of renal fibrosis Nephrol Dial Transplant. 2004;19(7):1716-1723.

57. Gleeson MJ. Alport's syndrome: audiological manifestations and implications. J Laryngol Otol. 1984;98(5):449-465

58. Wester DC, Atkin CL, Gregory MC. Alport syndrome: clinical update. J Am Acad Audiol. 1995;6(1):73-79.

59. Flinter FA, Cameron JS, Chantler C, Houston I, Bobrow M. Genetics of classic Alport's syndrome. Lancet. 1988;2(8618):1005-1007.

60. Gratton MA, Rao VH, Meehan DT, Askew C, Cosgrove D. Matrix metalloproteinase dysregulation in the stria vascularis of mice with Alport syndrome: implications for capillary basement membrane pathology. Am J Pathol. 2005;166(5):1465-1474.

61. Weidauer H, Arnold W. [Morphological changes in the inner ear of alport's syndrome (author's transl)]. Laryngol Rhinol Otol (Stuttg). 1976;55(1):6-16

62. Kim HJ, et al. Patterns of gene expression associated with Pten deficiency in the developing inner ear. PLoS One. 2014;9(6):e97544.

63. Teekhasaenee C, et al. Posterior polymorphous dystrophy and Alport syndrome. Ophthalmology. 1991;98(8):1207-1215.

64. Herwig MC, Eter N, Holz FG, Loeffler KU. Corneal clouding in Alport syndrome. Cornea. 2011;30(3):367-370.

65. Streeten BW, Robinson MR, Wallace R, Jones DB. Lens capsule abnormalities in Alport's syndrome. Arch Ophthalmol. 1987;105(12):1693-1697.

66. Cheong HI, Kashtan CE, Kim Y, Kleppel MM, Michael AF. Immunohistologic studies of type IV collagen in anterior lens capsules of patients with Alport syndrome. Lab Invest. 1994;70(4):553-557.

67. Pei Z, et al. Osteopontin deficiency reduces kidney damage from hypercholesterolemia in Apolipoprotein E-deficient mice. Sci Rep. 2016;6:28882.

68. Rittling SR, Chambers AF. Role of osteopontin in tumour progression. Br J Cancer. 2004;90(10):1877-1881.

69. Rangaswami H, Bulbule A, Kundu GC. Osteopontin: role in cell signaling and cancer progression. Trends Cell Biol. 2006;16(2):79-87.

70. Sangaletti S, et al. Osteopontin shapes immunosuppression in the metastatic niche. Cancer Res. 2014;74(17):4706-4719.

71. Grgic I, et al. Targeted proximal tubule injury triggers interstitial fibrosis and glomerulosclerosis. Kidney Int. 2012;82(2):172-183.

72. Girard E, et al. The dynamin chemical inhibitor dynasore impairs cholesterol trafficking and sterol-sensitive genes transcription in human HeLa cells and macrophages. PLoS ONE. 2011;6(12):e29042.

73. Robinet $\mathrm{P}$, et al. Dynamin is involved in endolysosomal cholesterol delivery to the endoplasmic reticulum: role in cholesterol homeostasis. Traffic. 2006;7(7):811-823.

74. Che R, Yuan Y, Huang S, Zhang A. Mitochondrial dysfunction in the pathophysiology of renal diseases. Am J Physiol Renal 
Physiol. 2014;306(4):F367-F378.

75. Ashrafian $\mathrm{H}$, et al. A mutation in the mitochondrial fission gene Dnm11 leads to cardiomyopathy. PLoS Genet. 2010;6(6):e1001000

76. Rogers GW, et al. High throughput microplate respiratory measurements using minimal quantities of isolated mitochondria. PLoS One. 2011;6(7):e21746.

77. Kamiyama M, Garner MK, Farragut KM, Kobori H. The establishment of a primary culture system of proximal tubule segments using specific markers from normal mouse kidneys. Int J Mol Sci. 2012;13(4):5098-5111.

78. Ioannou GN, Haigh WG, Thorning D, Savard C. Hepatic cholesterol crystals and crown-like structures distinguish NASH from simple steatosis. J Lipid Res. 2013;54(5):1326-1334.

79. Diogo Benchimol de Souza BMG, Marlene Benchimol, Fernanda Amorim de Morais Nascimento. Evaluation of the glomerular filtration barrier by electron microscopy. In: Janecek M, ed. Modern Electron Microscopy in Physical Life Sciences. Rijeka, Croatia: InTechOpen:2016.

80. Yang X, et al. Tri-iodo-1-thyronine promotes the maturation of human cardiomyocytes-derived from induced pluripotent stem cells. J Mol Cell Cardiol. 2014;72:296-304. 